\title{
Numerical Study of Surface Pressure Fluctuation on Rigid Disk-Gap-Band-Type Supersonic Parachutes
}

\author{
K. Kitamura* and K. Fukumoto \\ Yokohama National University, Yokohama 240-8501, Japan \\ and \\ K. Mori主 \\ Nagoya University, Nagoya 464-8603, Japan \\ https://doi.org/10.2514/1.J059190
}

\begin{abstract}
In the aerodynamic characteristics of supersonic parachutes, it is important to understand surface pressure distribution because it is strongly related to the fluctuation of drag and problematic unstable deformation of a parachute. However, there is a paucity of studies that focuses on the detailed surface pressure distribution. Therefore, we investigated the interior and exterior of a rigid disk-gap-band-type parachute as the first step, under the assumption that the forebody or suspension lines are absent, and thus the pressure and drag fluctuations are small. Two configurations are considered: one with a continuous gap and a vent orifice, representing a conventional Disk-GapBand parachute, and one with a discontinuous gap made up of 8 separate orifices and a vent orifice. By making the gap discontinuous, the interior and exterior pressure fluctuations are reduced. Furthermore, as indicated by the flowfield analysis, the discrete gap reduces the asymmetric pressure distribution interior the parachute, and the interior pressure fluctuation far from the center is suppressed. The result is considered useful for the suppression of unstable deformation such as area oscillation. This is currently a problem in supersonic parachute operation. In addition, we have identified locations on the model surface where the pressure fluctuations contribute to the drag fluctuations of the model.
\end{abstract}

\begin{tabular}{|c|c|c|}
\hline \multicolumn{3}{|r|}{ Nomenclature } \\
\hline$a$ & $=$ & speed of sound \\
\hline$C_{D}$ & $=$ & drag coefficient \\
\hline$C_{p}$ & $=$ & pressure coefficient \\
\hline$D_{p}$ & $=$ & projected diameter of canopy \\
\hline$D_{V}$ & $=$ & vent diameter \\
\hline$D_{0}$ & $=$ & nominal (reference) diameter of canopy \\
\hline$e_{T}$ & $=$ & total energy per unit volume \\
\hline $\boldsymbol{E}_{k}, \boldsymbol{F}_{k}$ & $=$ & $\begin{array}{l}\text { inviscid and viscous flux vectors in } k \text { direction }(k= \\
1,2,3 \text { corresponding to } x, y, z \text {, respectively) }\end{array}$ \\
\hline$H$ & $=$ & total enthalpy \\
\hline $\boldsymbol{H}_{B}$ & $=$ & height of band \\
\hline$H_{C}$ & $=$ & height of canopy \\
\hline$H_{G}$ & $=$ & height of gap \\
\hline$M$ & $=$ & Mach number \\
\hline $\mathrm{Pr}$ & $=$ & Prandtl number; 0.71 \\
\hline$p$ & $=$ & local pressure \\
\hline$p_{0}$ & $=$ & total pressure \\
\hline$Q$ & $=$ & conservative variable vector \\
\hline$q$ & $=$ & $\begin{array}{l}\text { primitive variable }(\rho, u, v, w \text {, or } p \text { in three dimen- } \\
\text { sions) }\end{array}$ \\
\hline$R$ & $=$ & reattachment region \\
\hline$R e$ & $=$ & $\begin{array}{l}\text { Reynolds number based on } 1 \mathrm{~m} \text { and freestream speed } \\
\text { of sound }\end{array}$ \\
\hline$R e_{D p}$ & $=$ & Reynolds number based on $\boldsymbol{D}_{p}$ and freestream velocity \\
\hline$S_{\text {opening }}$ & $=$ & total opening area \\
\hline$S_{G}$ & $=$ & gap opening area \\
\hline$S_{p}$ & $=$ & projected area of canopy \\
\hline$S_{R}$ & $=$ & separated region \\
\hline
\end{tabular}

Received 25 October 2019; revision received 29 May 2020; accepted for publication 27 July 2020; published online 11 November 2020. Copyright (C) 2020 by the American Institute of Aeronautics and Astronautics, Inc. All rights reserved. All requests for copying and permission to reprint should be submitted to CCC at www.copyright.com; employ the eISSN 1533-385X to initiate your request. See also AIAA Rights and Permissions www.aiaa.org/randp.

*Associate Professor, Graduate School of Engineering, 79-5 Tokiwadai, Hodogaya-ku, Kanagawa. Senior Member AIAA.

${ }^{\dagger}$ Graduate Student, Graduate School of Engineering, 79-5 Tokiwadai, Hodogaya-ku, Kanagawa.

${ }^{\ddagger}$ Associate Professor, Graduate School of Engineering, Furouchou, Chikusa-ku, Aichi.

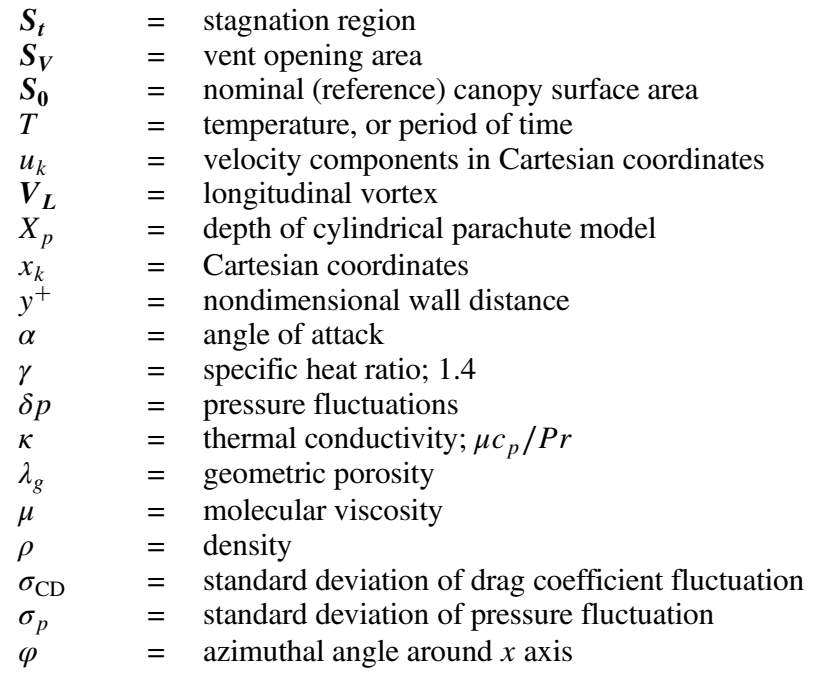

\section{Subscripts}

$\begin{array}{ll}\text { ave } & =\text { average value } \\ \text { case1 } & =\text { value of case } 1 \\ \text { case2 } & =\text { value of case } 2 \\ i & =\text { cell } i \\ i, j & =\text { interface between cells } i \text { and } j \\ \text { in } & =\text { value inside canopy } \\ \text { out } & =\text { value outside canopy } \\ 0 & =\text { value at azimuthal angle } \varphi=0 \\ 22.5 & =\text { value at azimuthal angle } \varphi=22.5 \\ \infty & =\text { freestream value }\end{array}$

\section{Introduction}

$\mathbf{R}$ ECENTLY in the space development field, manned planetary exploration (especially to Mars) attracted worldwide attention. To realize future manned planetary exploration, a more reliable parachute system is necessary for landing. The disk-gap-band (DGB)-type supersonic parachute is traditionally adopted [1] , and the parachute exhibits the open areas termed as the gap and vent (see Fig. 1). The flow of air from the open areas reduces the force generated during parachute 


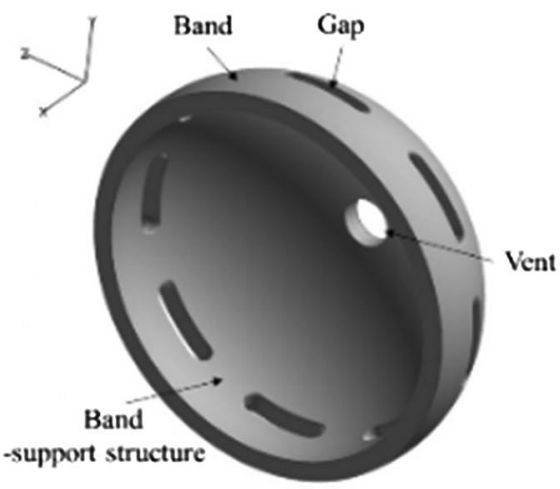

a) Case 1 (perspective view)

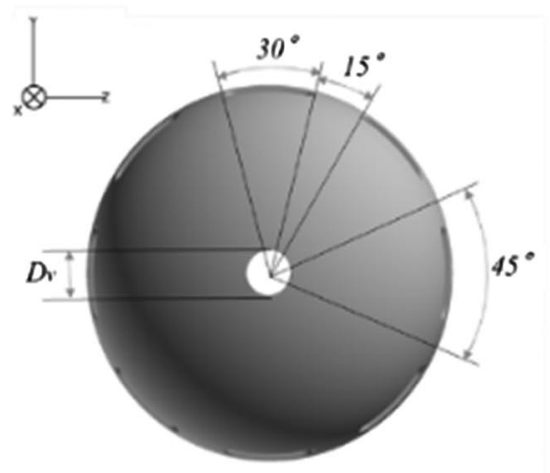

c) Case 1 (rear view)

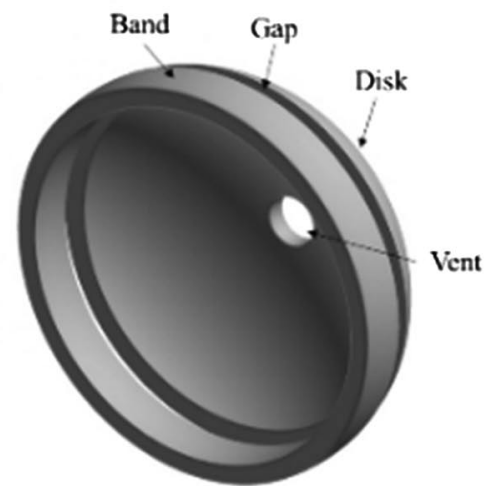

b) Case 2 (perspective view)

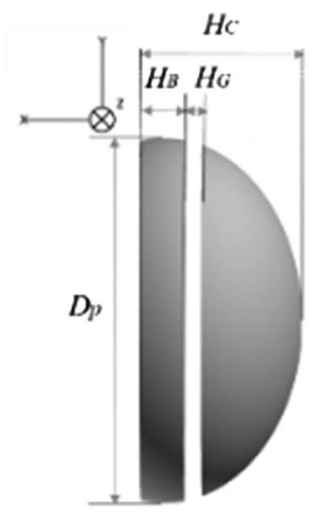

d) Case 2 (side view)

Fig. 1 Configuration.

inflation [2]. The supersonic parachute also exhibits significant advantages in terms of drag per weight and transportability, although its reliability still remains a challenge. The interaction between the wake of the payload and detached shock wave is focused on as an unstable phenomenon. With respect to the phenomenon, the conditions to avoid large-scale interference were clarified in several previous studies [3- $-\underline{5}$. Let us briefly review the related, earlier works (computational studies are summarized in Table 1).

Karagiozis et al. [6] conducted an elaborate ghost-fluid-methodbased large-eddy simulation (LES) on the Mach 2 flow around a DGBtype parachute, where the forebody and the parachute deformation were considered but permeability treatment was not clearly explained. In spite of their considerable effort, however, their solution still exhibits $20 \%$ errors compared with the corresponding experiment conducted by Sengupta et al. [7]. According to Karagiozis et al. [6], this difference arose from the poor resolution at the wake flow and the shock interaction. They also carried out the detached-eddy simulation, yielding only 3-6\% differences from the LES result. In addition, they actually focused on the canopy deformation itself but did not examine the detailed wake flow dynamics. Another important work by Kim and Peskin [8] simulated flows around single and multiple parachutes using the immersed-boundary method (IBM). However, their conditions were limited to very low speed $(0.6-1.2 \mathrm{~m} / \mathrm{s})$. Xue and Nakamura [9] and Xue et al. [10,11] conducted a series of studies on supersonic parachutes. In Refs. $[10,11]$, they assumed the parachute canopy was rigid (i.e., no deformation was considered) and focused on its surrounding supersonic flow physics, such as shock interactions and oscillations: Particular attention was paid to the separation between the forebody and the canopy in Ref. [10], whereas the effects of the (rigid-body-modeled) suspension lines were discussed in Ref. [11]. The effect of separation distance was surveyed in Ref. [9] for a flexible canopy. All these findings were of great importance, but they assumed laminar flows throughout their papers. They claimed that their solution was in good agreement with the experimental data for time-averaged surface pressure values. However, it is questionable whether turbulent effects were truly negligible in their cases, especially in terms of unsteadiness of the wake flow and the shock. Guruswamy [12] did three-degree-of-freedom (3-DOF) computations at Mach $0 . \overline{43}$ and 2.0. However, he employed a classical numerical flux (which has huge numerical dissipation) with a Reynolds-averaged Navier-Stokes (RANS) turbulence model. Hence, the resultant flow resolution could be somewhat degraded, although the method itself was worth mentioning. Tezduyar et al. [13] also conducted a milestone work. They proposed a fluid-structure-interaction (FSI)type method and applied it to a parachute canopy, considering its deformation, porosity, and the suspension lines. However, their flow velocity is limited to $25.7 \mathrm{ft} / \mathrm{s}$ (very low speed), which is obviously different from our target condition, i.e., an unsteady, supersonic

Table 1 Related computational parachute flow papers

\begin{tabular}{|c|c|c|c|c|c|c|c|}
\hline Authors & Primary focus & $\begin{array}{l}\text { Turbulent } \\
\text { treatment }\end{array}$ & $\begin{array}{c}\text { Mach } \\
\text { number }\end{array}$ & $\begin{array}{c}\text { Deformation } \\
\text { treatment }\end{array}$ & Permeability & Forebody & $\begin{array}{l}\text { Suspension } \\
\text { lines/risers }\end{array}$ \\
\hline Present & Wake/canopy & DDES & 2 & Rigid & No & No & No \\
\hline Karagiozis et al. [6] & Canopy deformation & LES & 2 & Ghost fluid & No & Yes & Yes \\
\hline Kim and Peskin $[\overline{8}]$ & Multiple parachutes & Laminar & $0.002-0.004$ & IBM & No & Yes & Yes \\
\hline Xue and Nakamura [9] & Canopy deformation & Laminar & $1.6-2.1$ & IBM & No & Yes & No \\
\hline Xue et al. [10] & Suspension lines & Laminar & 2 & Rigid/IBM & No & Yes & Yes \\
\hline Xue et al. $[\overline{11}]$ & Clearance & Laminar & 2 & Rigid & No & Yes & No \\
\hline Guruswamy [12] & Method & RANS & $0.43,2$ & 3-DOF/damper & No & Yes & No \\
\hline Tezduyar et al. [13] & Method & Laminar & 0.02 & FSI & Yes & No & Yes \\
\hline
\end{tabular}


turbulent wake flow. Moreover, the last two papers were dedicated to the proposed method therein and the demonstrations of their capabilities rather than the wake flow unsteadiness. This holds also to Stein et al.'s work [14], where fluid-structure-interaction (FSI) simulations were carried out on the parachute canopy with an apparently low flow velocity.

As indicated earlier in this paper, there is a paucity of extant studies on the supersonic DGB parachute's wake. The steady pressure distribution exterior to the canopy is examined with respect to a hemispherical cup [15] (which is a simpler form) and a flat circular ribbon parachute [16] (in which the influence on the wake is low due to a small opening area). However, the larger opening area of the DGB generates a larger jet, and the jet significantly interacts with the wake; this complicates the wake (the flow physics of such a jet formation and its shock interaction are very similar to those of crossflow jets, e.g., Refs. [17,18]). Simultaneously, the complicated wake can cause motion of the bow shock ahead of the canopy, possibly resulting in pressure changes inside the canopy and the area oscillation. To suppress the phenomenon, it is necessary to understand the basic physics of the flow around the DGB parachute. However, the wake of the DGB that is generated by the interaction between supersonic flow and a flexible body is extremely complicated [6]. To understand the complex flow, it is necessary to assume that there is no parachute deformation or no forebody (capsule ahead of the canopy). Furthermore, on the actual parachute surface, the interior and the exterior pressure fluctuations are closely related to the parachute deformation and flutter as previously described. However, it is difficult to investigate the wake in detail from the viewpoint of model support in a wind-tunnel test [7,19].

Hence, we will investigate the pressure fluctuation on the interior and exterior of a rigid model that mimics the supersonic DGB parachute by using computational fluid dynamics (CFD) as a first step to understanding the DGB wake: specifically, delayed detached-eddy simulation (DDES) [20], which captures most of the wake flows by accurate LES but switches to economical RANS at near-wall flows (and is now becoming one of the standard methods for afterbody flows [21]), will be used. Additionally, we will discuss the fundamental physics of the flow around a model representing a DGB canopy in isolation, including the relation between the surface pressure fluctuation amplitude and characteristics of the flowfield or position. Furthermore, we will select the gap configuration that significantly influences the flowfield as a parameter and compare the difference in the surface pressure fluctuation amplitude. We focus on the position that significantly influences parameters including the area oscillation and obtain a better gap configuration to reduce the pressure fluctuation there. It is expected that the present results will contribute to the realization of a more reliable planetary landing system as a fundamental survey excluding the complex canopy deformation and the forebody interaction.

\section{Numerical Setup}

\section{A. DGB Parachute Model Configuration}

In the study, we use two types of rigid models that mimic the DGB parachute to investigate the effect of the opening area configurations on the drag and the surface pressure distributions. Case 1 (Fig. 1a) mimics the rigid model used in the wind-tunnel test. In the rigid-model wind-tunnel tests [22], it is necessary to support the band. Thus, case 1 also has eight band-support structures at $45 \mathrm{deg}$ intervals. Thus, the gap of case 1 is separated and discretized by the structure. The thickness $(2 \mathrm{~mm})$ and the (rectangular) leading-edge configuration were decided according to manufacturing tolerances [22].

Next, case 2 (Fig. 1b) simulates a traditional and actual DGB parachute. However, in case 2, suspension lines between the disk and band are omitted because they are extremely thin (please see Refs. [6,10] for their influences). Thus, the gap in case 2 is continuous. This is the main difference between case 1 and case 2 .

The Cartesian coordinate system $(x, y, z)$ is defined such that its origin corresponds to the center of the leading edge of the parachutes. Specifically, the $x$ axis is along the direction of the central axis of the parachute, the $y$ axis is defined so that the angle of attack is defined around it, and the $z$ axis is orthogonal to the aforementioned two.
The key geometric parameters of the two types of DGB parachutes are shown in the following (Table 2, and Figs. 1c and 1d). All parameters (with the exception of the vent and related parameters) are consistent with the experiment [22]. In parachute development, the nominal (reference) area $S_{0}$ or the $x$-directional projected parachute surface area $S_{p}$ (including opening areas such as gaps and vents) is traditionally used to calculate the aerodynamic coefficients. The nominal (reference) diameter $D_{0}$, for instance, is given as follows:

$$
D_{0}=\sqrt{4 S_{0} / \pi}
$$

In Table 2 , all the key parameters normalized by $D_{0}$ or $S_{0}$ are shown in brackets.

Additionally, the geometric porosity $\lambda_{g}$ is an important parameter for supersonic parachute development. Specifically, $\lambda_{g}$ denotes the geometric opening area per total parachute surface area and is given as follows:

$$
\lambda_{g}=\frac{S_{\text {opening }}}{S_{0}}=\frac{\left(S_{G}+S_{v}\right)}{S_{0}}
$$

Based on Table 2, the geometric porosity of case $1 \lambda_{g \text {,case } 1}$ is $8.2 \%$ and that of case $2 \lambda_{g \text {,case } 2}$ is $11.9 \%$. For reference purposes, the geometric porosity of the Mars Science Laboratory is approximately $12.8 \%$ [23]. In terms of the geometric porosity, the parameter that is presently used is different because our DGB parachute configuration mimics a wind-tunnel experiment model as previously described. Thus, this is not a problem. Our motivation involves understanding basic flow physics by comparing computational results with the wind-tunnel experimental results.

\section{B. Governing Equations}

In the study, the governing equations are three-dimensional compressible Navier-Stokes equations as follows [Eqs. $(\underline{3 a-3 c})]$ :

$$
\begin{gathered}
\frac{\partial Q}{\partial t}+\frac{\partial F e_{k}}{\partial x_{k}}=\frac{1}{R e} \frac{\partial F v_{k}}{\partial x_{k}} \\
Q=\left(\begin{array}{c}
\rho \\
\rho u_{l} \\
e_{T}
\end{array}\right), \quad \boldsymbol{F e}=\left(\begin{array}{c}
\rho u_{k} \\
\rho u_{l} u_{k}+p \delta_{l k} \\
\left(e_{T}+p\right) u_{k}
\end{array}\right), \\
F v=\left(\begin{array}{c}
0 \\
\tau_{l k} \\
u_{m} \tau_{m k}+\frac{\kappa}{(\gamma-1) \operatorname{Pr}} \frac{\partial T}{\partial x_{k}}
\end{array}\right)
\end{gathered}
$$

Table 2 Key geometric parameters of the DGB parachute

\begin{tabular}{lc}
\hline \hline Parameter & Value \\
\hline Nominal (reference) diameter $D_{0}, \mathrm{~mm}$ & 53.8 \\
Projected diameter $D_{p}, \mathrm{~mm}$ & 40.0 \\
{$\left[D_{p} / D_{0}\right]$} & {$[0.744]$} \\
Vent diameter $D_{v}, \mathrm{~mm}$ & 4.90 \\
{$\left[D_{p} / D_{0}\right]$} & {$[0.091]$} \\
Band height, $H_{B}(\mathrm{~mm})$ & 4.83 \\
{$\left[H_{B} / D_{0}\right]$} & {$[0.090]$} \\
Gap height $H_{G}, \mathrm{~mm}$ & 2.00 \\
{$\left[H_{G} / D_{0}\right]$} & {$[0.037]$} \\
Canopy height $H_{C}, \mathrm{~mm}$ & 17.5 \\
{$\left[H_{C} / D_{0}\right]$} & {$[0.325]$} \\
Nominal (reference) area $S_{0}, \mathrm{~mm}^{2}$ & $2.27 \times 10^{3}$ \\
Projected area, $S_{p}, \mathrm{~mm}{ }^{2}$ & $1.26 \times 10^{3}$ \\
{$\left[S_{p} / S_{0}\right]$} & {$[0.553]$} \\
Gap areas $S_{G, \text { case1 }}$ and $S_{G, \text { case } 2}, \mathrm{~mm}^{2}$ & $1.67 \times 10^{2}, 2.51 \times 10^{2}$ \\
{$\left[S_{G, \text { case1 }} / S_{0}, S_{G, \text { case } 2} / S_{0}\right]$} & {$[0.074,0.111]$} \\
Vent area $S_{v}$, mm $^{2}$ & $1.89 \times 10^{1}$ \\
{$\left[S_{v} / S_{0}\right]$} & {$[0.0083]$} \\
Geometric porosities $\lambda_{g, \text { case1 }}$ and $\lambda_{g, \text { case2 }}, \%$ & $8.2,11.9$ \\
\hline \hline
\end{tabular}




$$
\tau_{j k}=\mu\left(\frac{\partial u_{j}}{\partial x_{k}}+\frac{\partial u_{k}}{\partial x_{j}}\right)-\frac{2}{3} \mu \frac{\partial u_{l}}{\partial x_{l}} \delta_{j k}
$$

where subscripts $k, l, m$, and $n$ correspond to 1,2 , and 3 denoting the Cartesian coordinates; $\rho$ denotes the density; $u_{i}$ denotes the velocity components $(i=1,2$, and 3 corresponding to $u, v$, and $w$, respectively); $e_{T}$ denotes the total energy per unit volume; $p$ denotes the pressure; $H$ denotes the total enthalpy $\left[H=\left(e_{T}+p\right) / \rho\right]$; and $T$ denotes the temperature. The working gas is air, which is approximated by the calorically perfect gas model with the specific heat ratio of $\gamma=1.4$. The Prandtl number is $\operatorname{Pr}=0.71$. The molecular viscosity $\mu$ as calculated via Sutherland's formula and thermal conductivity $\kappa$ are related as $\kappa=c_{p} \mu / P r$, where $c_{p}$ denotes the specific heat at constant pressure. To incorporate the turbulence effects, the molecular viscosity $\mu$ is replaced by $\left(\mu+\mu_{t}\right)$, where $\mu_{t}$ denotes the turbulence viscosity. Similarly, $\kappa$ is replaced by $\left(\kappa+c_{p} \mu_{t} / P r_{t}\right)$, and $P r_{t}$ denotes the turbulent Prandtl number corresponding to 0.90 .

The Reynolds number is defined in Eq. (4) as follows:

$$
R e=\frac{\rho_{\infty} a_{\infty} L_{\infty}}{\mu_{\infty}}
$$

where $L_{\infty}$ is a unit length $(1 \mathrm{~m})$, and $a_{\infty}$ denotes a freestream sound speed. Note that this Reynolds number appears as a result of normalization of the Navier-Stokes equations, whereas $U_{\infty}$ (a freestream velocity) may be used as a reference velocity when it comes to the experimental flow conditions, for instance.

\section{Computational Methods}

We conduct three-dimensional numerical computation by using Fast aerodynamic routines (known as FaSTAR) [24] developed at the Japan Aerospace Exploration Agency (JAXA) as a high-speed flow solver for unstructured grids. The system of equations [Eq. (3)] is discretized via a cell-centered finite volume method. We use DDES [20] based on the Spalart-Allmaras one-equation model with no tripping term $f_{t 2}$ with rotation correction (SA-noft2-R) as the turbulence model. This corresponds to a Reynolds-averaged Navier-Stokes/ large-eddy simulation hybrid model. In the model, a near-wall region is modeled via SA-noft2-R (RANS family model), and the other regions are modeled via LES. As a result, each computational case can be completed within a few weeks at JAXA's second-generation supercomputer system. A numerical flux for an inviscid term is calculated by an advection upstream splitting method (AUSM)-type scheme, namely, simple low-dissipation AUSM [25]. The Green-Gauss-based weighted least-squares method [26] is used for gradient reconstruction with a Venkatakrishnan slope limiter [27]. With respect to the time integration method, a lower-upper symmetric Gauss-Seidel scheme [28] is adopted with a second-order backward difference.

\section{Computational Grids}

We use HexaGrid [29] (which is an automatic meshing tool developed at JAXA) to generate the unstructured computational grids based primarily on hexahedral Cartesian grids (that are not axisymmetric and will play a role in breaking the flow symmetry). The computational grids for case 1 and case 2 are shown in Figs. $2 \mathrm{a}$ and $2 \mathrm{~b}$, respectively. The coordinate system is defined as mentioned earlier in this paper. As shown in Fig. 2c, the computational domain corresponds to a $35 D_{0} \times 35 D_{0}$ cube that is filled with hexahedra, prisms, pyramids, and tetrahedra; and the origin of the coordinate system (i.e., the parachute nose) is set at $8.75 D_{0}$ downstream from the inflow boundary. Furthermore, the number of cells is approximately $8.6 \times 10^{6}$ for each case. To resolve the boundary layer, the first cell height is defined to satisfy $y^{+}<1$. Furthermore, the confirmation of grid convergence will be described in Sec. III.B.

\section{E. Computational Conditions}

The computational conditions are selected to correspond to the wind-tunnel test [22] conditions in Table $\underline{3}$, such as the freestream
Mach number $M_{\infty}=2.0$, Reynolds number $R e_{D p}$ (based on the diameter of the body), total pressure $p_{0}$, and local pressure $p_{\infty}$ :

$$
R e_{D_{p}}=\frac{\rho_{\infty} U_{\infty} D_{p}}{\mu_{\infty}}
$$

Additionally, the angle of attack throughout the study corresponds to $0 \mathrm{deg}$.

\section{F. Aerodynamic Coefficients}

We defined the axial force coefficient and the pressure coefficient $C_{p}$ as follows:

$$
\begin{gathered}
C_{D}=\frac{F_{x}}{q_{\infty} S_{0}} \\
C_{p}=\frac{p-p_{\infty}}{q_{\infty}}
\end{gathered}
$$

where $F_{x}$ denotes the x component force; $p$ denotes local pressure; and $p_{\infty}$ and $q_{\infty}$ denote freestream pressure and dynamic pressure, respectively.

\section{G. Pressure Measurement Point Distribution}

We set 20 pressure measurement points on the surface of the parachute models to investigate pressure fluctuations. As shown in Fig. 3 , 10 points were set on the exterior of the parachute, and the other 10 points were set on the interior of the parachute. The aim of the setting involves comparing the amount of exterior pressure fluctuations of the parachute with those interior the parachute. As shown in Fig. $\underline{3}$, measurement points $1-5$ are set at the positions where the distance from the center corresponds to $20,40,60,80$, and $92.5 \%$ of the radius. The points are labeled with a subscript of "in" or "out" that denotes the interior or exterior of the parachute, respectively. Additionally, the points are also labeled with a subscript of " 0 " or "22.5" that denotes the azimuthal angle $\varphi$ (see Fig. 3 ) around the $x$ axis (center axis of the parachute) to investigate the effect of the band-support structure on pressure fluctuations. The point sequence of $1_{0}-5_{0}$ is set in the range of the azimuthal angle of the "gap influence region," and the point sequence of $1_{22.5}-5_{22.5}$ is set in the range of the azimuthal angle of the "no gap influence region." For example, the measurement point that is termed as $1_{\text {out } 0}$ denotes that the point at which the distance from the center corresponds to $20 \%$ of the radius exterior, and its azimuthal angle $\varphi=0$.

\section{Results and Discussions}

\section{A. Validation}

First, we conduct computations for two different parachute configurations to validate our computational methods and setup. A configuration (case 1) corresponds to the comparison in terms of the exterior surface flowfield of the parachute. Another configuration (a cylindrical parachute without gaps) is in terms of the interior pressure fluctuation of the parachute.

\section{Surface Flowfield Validation for Unsteady Computations}

To validate our unsteady computations, we compare a timeaveraged solution of our unsteady computation with the final result of an oil flow experiment [22] that is assumed to capture a global and large-scale (low-frequency) surface flowfield. We create the averaged data by using 100 files with 500 time-step intervals (i.e., averaged over every $0.0935 \mathrm{~s}$ ). Therefore, the total average section is $9.35 \mathrm{~s}$. Figure 4 shows both computational and experimental exterior surface flowfields of the DGB parachute model. As shown in the figures, our computational result is in qualitative agreement with the experimental result [22] in terms of the following: 1) patterns of origin of longitudinal vortex $\mathrm{V} 1,2$ ) patterns of the reattachment line of the horseshoe vortex, and 3) patterns of reverse flow.

We discuss the aforementioned three patterns. Initially, we focus on pattern 1 . The patterns are generated at both ends of each single 


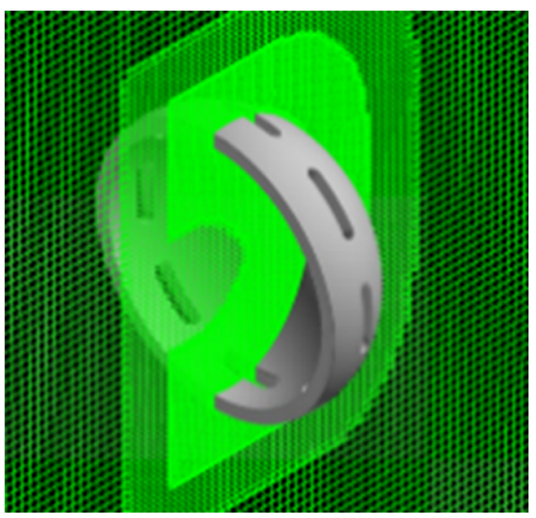

a) Case 1 (perspective view)

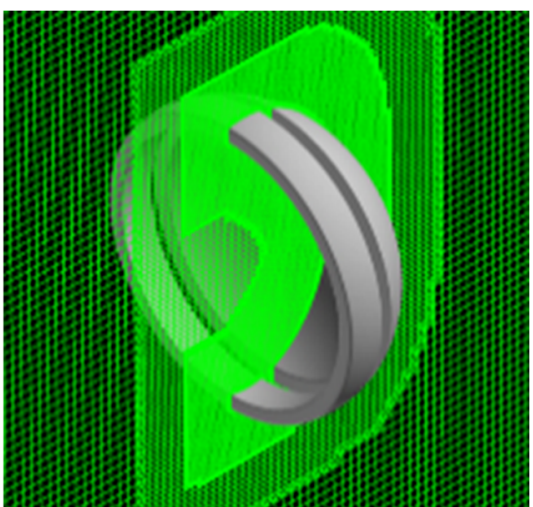

b) Case 2 (perspective view)

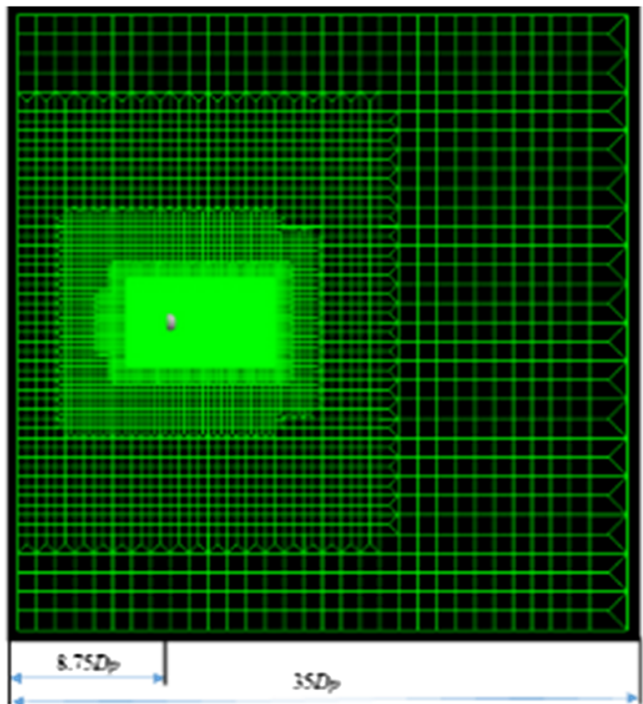

c) Overview

Fig. 2 Computational grid.

Table 3 Conditions of the supersonic wind-tunnel test (computation)

\begin{tabular}{lc}
\hline \hline$M_{\infty}$ & 2.0 \\
\hline$R e_{D p}$ & $6.60 \times 10^{5}$ \\
$p_{0}, \mathrm{kPa}$ & $1.32 \times 10^{2}$ \\
$p_{\infty}, \mathrm{kPa}$ & $1.71 \times 10^{1}$ \\
$T_{\infty}, \mathrm{K}$ & 177.78 \\
\hline \hline
\end{tabular}

gap hole, and we considered this as generated by the longitudinal vortex $V_{L}$ (see Fig. 5). Pattern 2 is termed as the reattachment line of the horseshoe vortex. Additionally, in the computational result, the line is closer to the gap hole than the experimental result. We assume that the difference is made by the turbulence model we used (SA-noft2-R-based DDES). In the model, regions near the wall are calculated by the SA-noft2-R RANS model. The model tends to overestimate the horseshoe vortex. In this case, a possibility exists wherein the primary vortex of the horseshoe vortex can be

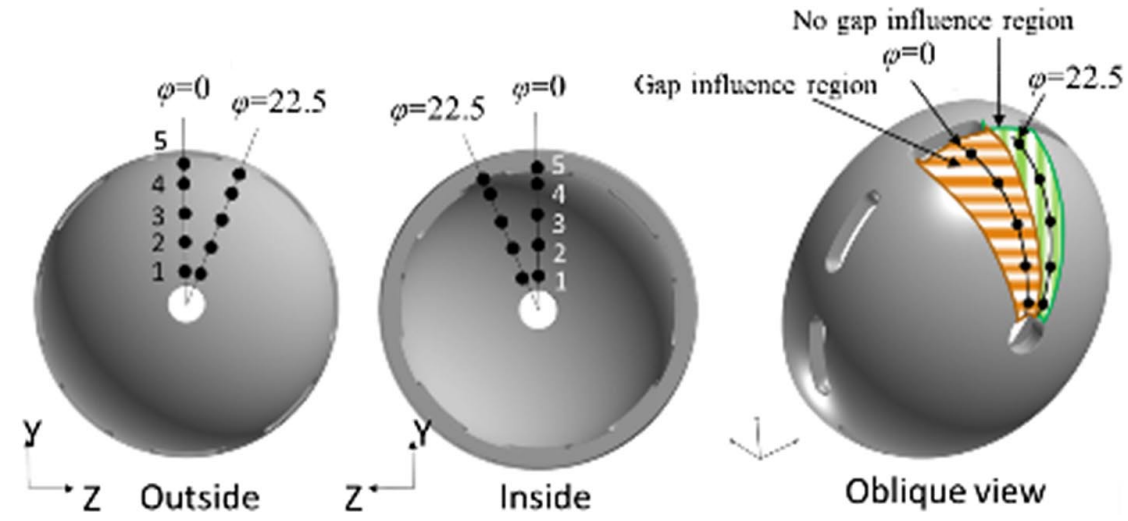

Fig. 3 Pressure measurement point distribution. 


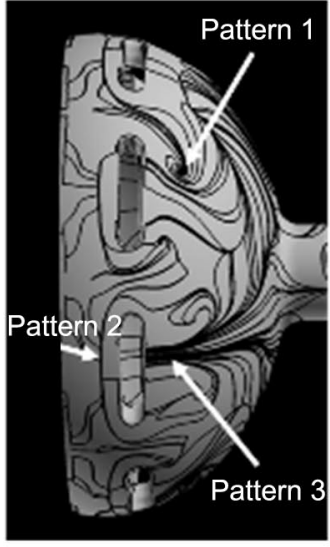

a) Surface flowfield of the computation (case 1)

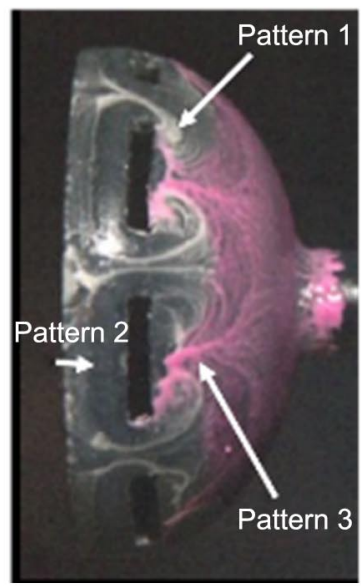

b) Surface flowfield of the oil flow experiment [22]

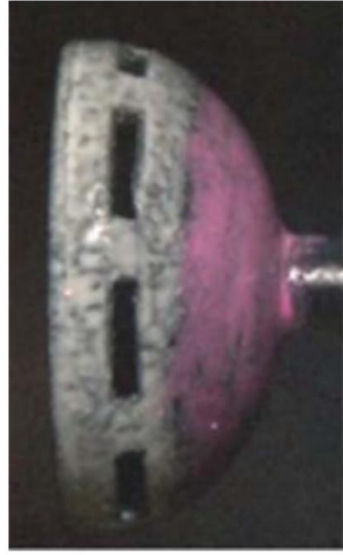

c) Initial condition of the oil flow experiment [22]

Fig. 4 Surface flowfield validation.

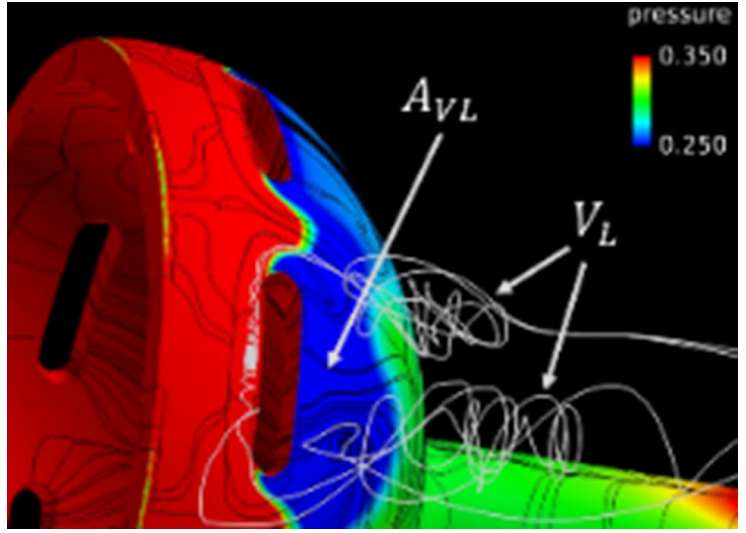

Fig. 5 Longitudinal vortex behind the gap (colored by static-pressurenormalized pressure $p / p_{\infty}$ instead of $C_{p}$ ).

overestimated (a pure LES may improve this, but at an expense of tremendous cost, which is beyond the scope of the present work). Finally, the patterns of reverse flow (pattern 3 ) are observed in both results. In the experiment, the downstream-half part corresponding to the exterior of the model is colored by pink oil (see Fig. 4c). Therefore, the forward movement of pink oil in Fig. $4 \mathrm{~b}$ represents the reverse flow.

\section{Pressure Fluctuation Validation for Unsteady Computations}

To validate our unsteady computational method, we compare the pressure fluctuations in our unsteady computation result with the fluctuations in the wind-tunnel test result that used a more simplified, cylindrical parachute model (Fig. 6 [ [ $]$ ]). The pressure measurement point is set at the position wherein the distance corresponds to half of the depth $X_{d}$ of the model. The position of the pressure measurement point corresponds to that in the experiment [5]. Figure 7 shows the result of the comparison. The computational result (orange-colored dashed line) captures two characteristics of the experimental result. The first characteristic is that the experimental result corresponds to $\delta p$ in the range of $100-400 \mathrm{~Hz}$. The other characteristic is that it is constant in the ranges of 10-100 and 400-1000 Hz. Therefore, we observed that our computational method sufficiently captures the characteristics of pressure fluctuations in the range of $10-1000 \mathrm{~Hz}$.

\section{B. Drag Coefficient Average and Fluctuations}

1. Unsteady Calculation and Average of Drag

An extremely important aspect of parachute performance is drag. Thus, we compare the drag coefficient $C_{D}$ of case 1 with that of

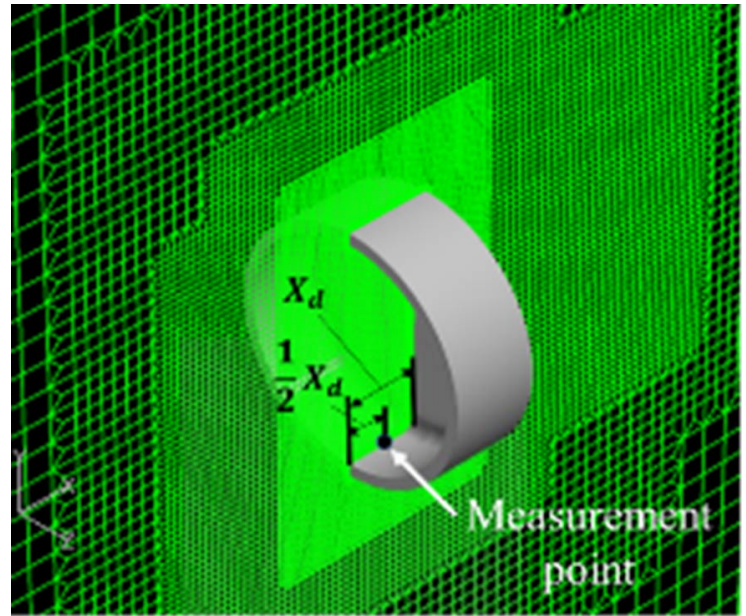

Fig. 6 Simplified cylindrical parachute model.

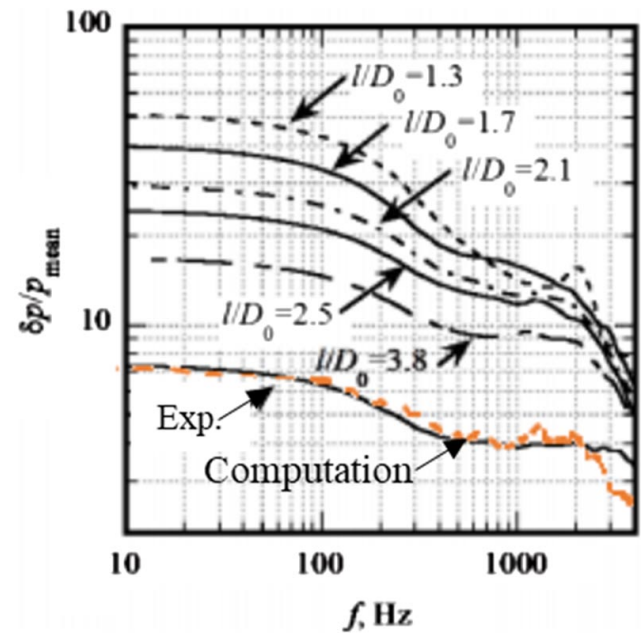

Fig. 7 Spectrums of interior pressure fluctuations of the parachute model [ $\underline{5}$ ].

case 2. The unsteady computation is conducted in two stages as shown in the following:

1) The first stage (preparation) has 30,000 time steps of unsteady calculation with $\Delta t=7.5 \times 10^{-5} \mathrm{~s}$ [maximum Courant-FriedrichsLewy $($ CFL) $\approx 1900]$. 
2) For the second stage (data acquisition), after the first-stage calculation, a 60,000-time-step unsteady calculation with $\Delta t=7.5 \times$ $10^{-5} \mathrm{~s}(\mathrm{CFL} \approx 1900)$ is conducted.

To eliminate the effect of the initial condition and to obtain the original unsteadiness, the data obtained from second-stage calculation were adopted. In the range of time, initially, we compared the average of $C_{D}\left(C_{D \text {,ave }}\right)$, as shown in Table 4 . The average is conducted by using the second-stage data. Additionally, $C_{D \text {, ave }}$ in case 1 is 0.928 , and case 2 is 0.918 ; i.e., the $C_{D \text {,ave }}$ in case 1 is $1.1 \%$ higher than that in case 2 . These results are obtained by using a "medium" computational grid in which a grid convergence was confirmed [see Fig. 8]. The result shown in Fig. 8 indicate that the medium grid is enough to eliminate the influence of grid resolution on $C_{D \text {,ave }}$. Herein, the cell number $N$ of each grid is shown in Table $\underline{5}$. For reference, there is the shape and the drag coefficients of another rigid DGB parachute model (having one-half of an ellipsoidal configuration, made of nylon, with the undisclosed geometric porosity $\lambda_{g}$ ) for another wind-tunnel test [30]. The $C_{D \text {,ave }}$ of this model is 0.88 , and this value is very similar to those obtained in the present study. The slight discrepancies from case 1 or case 2 would have come from the differences in the curvatures of the disk region and the geometric porosity $\lambda_{g}$.

\section{Fluctuation Amount of Drag}

Second, we also calculate the standard deviation of the fluctuation of $C_{D}\left(\sigma_{\mathrm{CD}}\right)$ to quantitatively compare the fluctuation amount of the cases. As shown in Table $4, \sigma_{\mathrm{CD}}$ of case 1 was $5.72 \times 10^{-4}$, and $\sigma_{\mathrm{CD}}$ of case 2 was $14.8 \times 10^{-4}$. Thus, the results indicate that the drag fluctuation amount of case 1 is approximately $60 \%$ smaller than that of case 2 . In terms of the two indices, case 1 may be preferable as a decelerator for use at Mach 2 because it exhibits the characteristics of

Table 4 Average and standard deviations of the $C_{D}$ fluctuation $\left(C_{D, \text { ave }}, \sigma_{\mathrm{CD}}\right)$

\begin{tabular}{llc}
\hline \hline & $C_{D, \text { ave }}$ & $\sigma_{\mathrm{CD}}$ \\
\hline Case 1 & 0.928 & $5.72 \times 10^{-4}$ \\
Case 2 & 0.918 & $14.8 \times 10^{-4}$ \\
\hline \hline
\end{tabular}

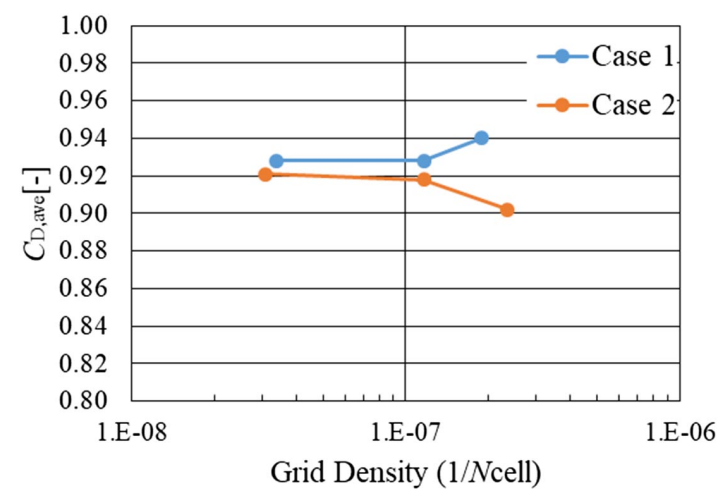

Fig. 8 Grid convergence confirmation.

Table 5 Cell number of each grid

\begin{tabular}{lcc}
\hline \hline & Case $1\{N$ cells $(1 / N)\}$ & Case $2\{N$ cells $(1 / N)\}$ \\
\hline Coarse & $5.3 \times 10^{6}\left(1.9 \times 10^{-7}\right)$ & $4.2 \times 10^{6}\left(2.4 \times 10^{-7}\right)$ \\
Medium & $8.6 \times 10^{6}\left(1.2 \times 10^{-7}\right)$ & $8.6 \times 10^{6}\left(1.2 \times 10^{-7}\right)$ \\
Fine & $29.6 \times 10^{6}\left(3.4 \times 10^{-8}\right)$ & $32.3 \times 10^{6}\left(3.1 \times 10^{-7}\right)$ \\
\hline \hline
\end{tabular}

high drag force and low drag fluctuation amount, although both of those fluctuations are very small compared with the average values.

\section{Frequency Component of Drag Fluctuation}

Third, in the range of time, we conduct a Fourier transform on the data of drag fluctuations to clarify major frequency components involved in the fluctuation. The results are shown in Fig. 9 as power spectral density (PSD) dual logarithmic graphs. In the graphs, the orange-colored curve shows the original data of the PSD. Furthermore, the blue-colored curve shows the simple moving average (SMA) of the original data to indicate the rough trends of original data. Subsequently, adjacent 20 data are used to conduct the SMA. Figure 9a shows the PSD of case 1 . The results indicated that the PSD decreases almost quadratically until approximately $18 \mathrm{~Hz}$, and thereafter decreases nearly linearly with respect to the frequency. Figure $9 \mathrm{~b}$ shows the PSD of case 2. The characteristic of the graph is that the PSD in the range of $10-160 \mathrm{~Hz}$ is especially high when compared to that in case 1 . The reason for this will be discussed in detail in Sec. III.E.

\section{Surface Pressure Fluctuations for the Interior and Exterior}

1. Exterior and Interior Surface Pressure Fluctuation Amounts

We focus on the surface pressure fluctuations interior and exterior each case. A surface pressure fluctuation is important because it involves deformation of the parachutes. We focus on 20 pressure measurement points, and their distributions are shown in Fig. 3. Based on the data obtained from the points, we calculate the standard deviation of the pressure fluctuation to evaluate the amount of fluctuations quantitatively. Table $\underline{6}$ shows the average of standard deviations of pressure fluctuations $\sigma_{p \text {,ave }}$. As shown in the table, in case 1 , the $\sigma_{p \text {,ave }}$ exterior is approximately five times that on the interior. Therefore, the outer pressure fluctuation is dominant in case 1. Conversely, in case 2 , the $\sigma_{p \text {, ave }}$ exterior is only 1.2 times that on the interior. Therefore, the results indicate that the interior pressure fluctuation is significant when compared with that in case 1 . Specifically, the pressure fluctuation of interior case 2 is 9.6 times that in case 1. Furthermore, the pressure fluctuation of exterior case 2 is approximately 2.3 times that of case 1 . (Thus, in case 2 , the gap configuration has reduced the interior pressure fluctuation by approximately $90 \%$ and the exterior pressure fluctuation by $57 \%$.) Based on the results, pressure and drag fluctuations are significantly underestimated while using the gap shape (case 1) that is tested on the wind tunnel.

\section{2. $\sigma_{p}$ of Each Pressure Measurement Point}

The next topic corresponds to the $\sigma_{p}$ of each pressure measurement point. Figures $10 \mathrm{a}$ and $10 \mathrm{~b}$ show the $\sigma_{p}$ on the exterior and interior, respectively, in case 1 . Figures $10 \mathrm{c}$ and $10 \mathrm{~d}$ show the $\sigma_{p}$ on the

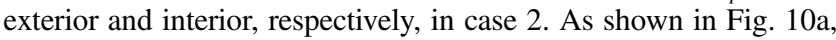
while the $\sigma_{p}$ of measurement point 1 is highest at $\phi=0\left(1_{\text {out } 0}\right)$, the $\sigma_{p}$ of measurement point 3 is highest at $\varphi=22.5\left(3_{\text {out } 22.5}\right)$. As shown in the result, the $\sigma_{p}$ on exterior case 1 exhibits a phase difference due to the influence of the discretized gap. The mechanism of the large fluctuation on the $3_{\text {out } 22.5}$ is explained later in Sec. III.E. Conversely, as shown in Fig. 10c, the $\sigma_{p}$ of measurement point 2 is highest at each azimuthal angle $\bar{\varphi}\left(2_{\text {out } 0}, 2_{\text {out } 22.5}\right)$. The mechanism of the large fluctuation on $2_{\text {out } 0}$ is also explained later in Sec. III.E. As shown in the figure, the magnitude of the $\sigma_{p}$ varies only based on the radial distance, and its magnitude correlation is the same in each azimuthal angle $\varphi$ because of the absence of the model shape difference between the azimuthal angles of 0 and 22.5. As shown in Fig. $10 \mathrm{~d}$, the $\sigma_{p}$ of measurement point 4 is the highest at each azimuthal angle $\varphi$; similarly, the magnitude correlation of $\sigma_{p}$ is the same in each azimuthal angle $\varphi$.

Conversely, it is known that an area oscillation (which corresponds to a problematic instability phenomenon of a supersonic parachute) occurs when the region far from the center is deformed [6]. Therefore, in order to suppress the area oscillation, it is considered as effective to reduce the pressure fluctuation in the region far from the center, such as measurement points 4 and 5 in the study. Figure $10 \mathrm{e}$ shows 


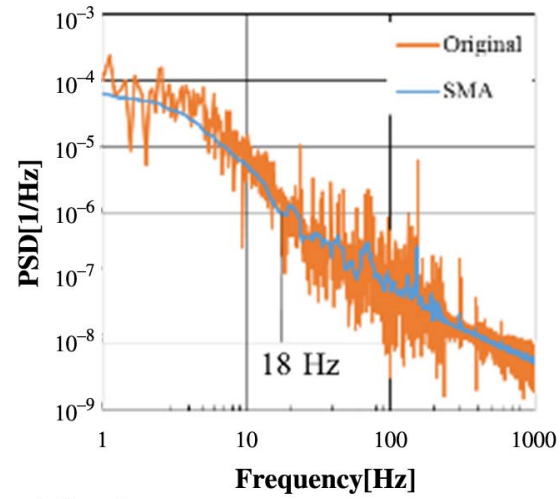

a) Case 1

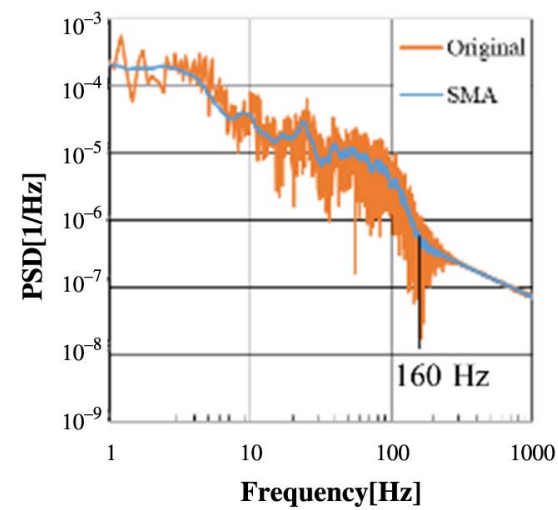

b) Case 2

Fig. 9 PSD graphs of $C_{D}$ fluctuations.

\begin{tabular}{lcc}
$\begin{array}{c}\text { Table } 6 \\
\text { of the pressure fluctuation }\left(\boldsymbol{\sigma}_{\boldsymbol{p} \text {,ave }}\right)\end{array}$ & $\begin{array}{c}\text { Average of the standard deviations } \\
\text { pxurer }\end{array}$ \\
\hline \hline & Exterior & Interior \\
\hline Case 1 & $5.83 \times 10^{-3}$ & $1.19 \times 10^{-3}$ \\
Case 2 & $13.6 \times 10^{-3}$ & $11.5 \times 10^{-3}$ \\
\hline \hline
\end{tabular}

the result of extracting $\sigma_{p}$ of measurement points 4 and 5 in each case. A comparison with case 2 indicates that the decrease in $\sigma_{p}$ interior case 1 is significant. The concrete mechanism for this will be explained subsequently in Sec. III.E. Furthermore, the sum of the $\sigma_{p}$ of measurement points 4 (interior and exterior) and 5 (interior and exterior) in case 1 is reduced by approximately $70 \%$ when compared to that in case 2 (see Fig. 10f). Therefore, case 1 (which exhibits a more complex configuration) appears to be better in terms of area oscillation suppression. On the actual, flexible parachute surface, the interior and the exterior pressures mutually interact and different modes might be activated. In such a case, the location of the pressure maximum might be different, and a parametric study taking the model structure into account will be necessary for stability analysis. But, still, the present findings will be useful pieces of information for fundamental understanding of the unsteady pressure fluctuations and their effects on the parachute deformation. Moreover, the present results directly apply to the early stages of the parachute development in which a rigid model is widely used.

\section{Relation Between Surface Pressure Fluctuations and Drag Coefficient Fluctuations}

In the previous section, we discussed the surface pressure fluctuations and drag coefficient fluctuations. In this section, we consider the part of the parachute that significantly contributes to the drag fluctuations by surveying the frequency component of each fluctuation.

\section{Case 1}

First, we focus on case 1, which exhibits a large pressure fluctuation on the exterior as mentioned in the previous section. As shown in Fig. 11a, the power spectral density graph of the drag fluctuation of case 1 superimposed on the PSD graphs of pressure measurement points $1_{\text {out22.5 }}$ and $3_{\text {out22.5. }}$. The points are selected because they exhibit the highest and second highest $\sigma_{p}$, and they adequately represent the frequency characteristics of the fluctuations in the surrounding points (see Figs. 11c and 11d). For example, in Fig. 11c, four PSD graphs of measurement points including $3_{\text {out } 22.5}$ are shown. These are located near the $3_{\text {out22.5 }}$, and these PSD graphs show almost identical trends. This indicates that these PSD graphs have different values but have similar frequency characteristics. Therefore, the frequency characteristics of $3_{\text {out } 22.5}$ represent its surrounding points, and they can attribute to the frequency characteristics of drag less than $15 \mathrm{~Hz}$. As seen in Fig. $11 \mathrm{a}$, for $15 \mathrm{~Hz}$ or less, the PSD of $3_{\text {out } 22.5}$ exhibits good agreement with the PSD of $C_{D}$. With respect to the PSD of $1_{\text {out } 22.5}$, on the other hand, it exhibits good agreement with the PSD of $C_{D}$ in the range of more than $15 \mathrm{~Hz}$. Thus, the drag fluctuation of less than $15 \mathrm{~Hz}$ is attributable to the position of $3_{\text {out } 22.5}$, and the fluctuation exceeding $30 \mathrm{~Hz}$ is attributable to $1_{\text {out } 22.5}$.

\section{Case 2}

Next, let us focus on case 2. As mentioned in the previous section, it is necessary to consider the interior and exterior pressure fluctuations. As shown in Fig. 11b, the PSD graph of the drag fluctuation in case 2 overlaps with the $\overline{\mathrm{PSD}}$ graphs of pressure measurement points $2_{\text {out } 0}$ and $4_{\text {in } 0}$. The PSDs of $2_{\text {out } 0}$ and $4_{\text {in } 0}$ are selected because they exhibit the highest $\sigma_{p}$ on the exterior and interior, respectively, and adequately represent the frequency characteristics of the fluctuation in the surrounding points (see Figs. $11 \mathrm{e}$ and $\underline{11 \mathrm{f}}$ ). Figure $11 \mathrm{e}$ indicates that these PSD graphs excluding $2_{\text {out } 0}$ show good agreement for $2_{\text {out } 0}$ of less than $20 \mathrm{~Hz}$. Therefore, $2_{\text {out } 0}$ can attribute the frequency characteristics of drag of less than $9 \mathrm{~Hz}$. In addition, Fig. $11 \mathrm{f}$ shows that these PSD graphs excluding $4_{\text {in } 0}$ exhibit good agreement for $4_{\text {in } 0}$ in the range of 20-200 Hz. As shown in Fig. 11b, the results indicate that the PSD of $2_{\text {out } 0}$ is consistent with the PSD of $C_{D}$ in the range of less than $9 \mathrm{~Hz}$. It was also observed that the PSD of $4_{\text {in } 0}$ exhibits good agreement with the PSD of $C_{D}$ in the range exceeding $100 \mathrm{~Hz}$. The agreements suggest that the drag fluctuation of less than $9 \mathrm{~Hz}$ mainly occurs at the position corresponding to $2_{\text {out } 0}$ and the fluctuation of more than $100 \mathrm{~Hz}$ is attributable to $4_{\text {in } 0}$.

As shown in the results for case 1 and case 2, the positions that significantly contribute to the drag fluctuation are identified. It is considered that the aforementioned positions would contribute to the future improvement of a conventional DGB parachute, at least for the present setup. Additionally, in each case, the results indicate that the $C_{D}$ fluctuation of the entire parachute can be represented by the PSD graph at only two points on its surface. These remarks will be verified by taking the forebody and the model support into account in future work.

\section{E. Relationship Between the PSD Graph and Flowfield}

In the previous section, we clarified the relationship between the fluctuation components of the pressure fluctuation and the position. In this section, we discuss how the pressure fluctuations are created by the flowfield.

\section{Case 1: Exterior}

Initially, we focus on case 1 . Figure 12 shows a period of variation in the pressure distribution of the $3_{\text {out } 22.5}$ in case 1 . The black lines on the surface of the parachute denote the surface streamline. As shown in Fig. 12, there are approximately four regions termed as area of the longitudinal vortex $A_{V L}$. $A_{V L}$ is generated by longitudinal vortices and barely moves. Therefore, the main pressure fluctuation by moving of the reattachment region occurs in the region, with the exception of $A_{V L}$ 


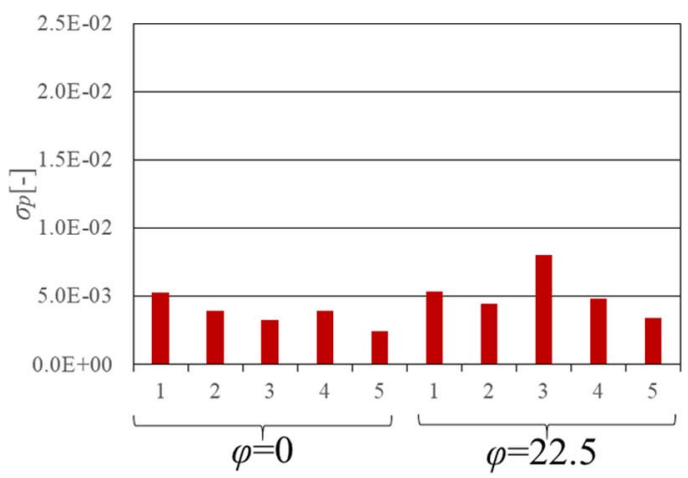

a) Case 1: exterior

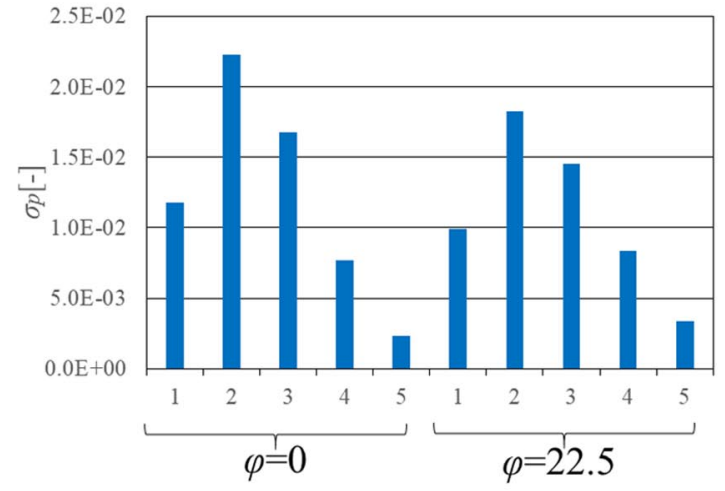

c) Case 2: exterior

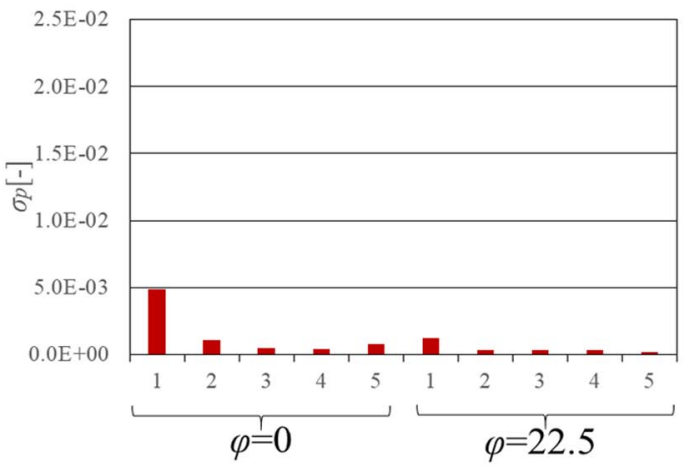

b) Case 1: interior

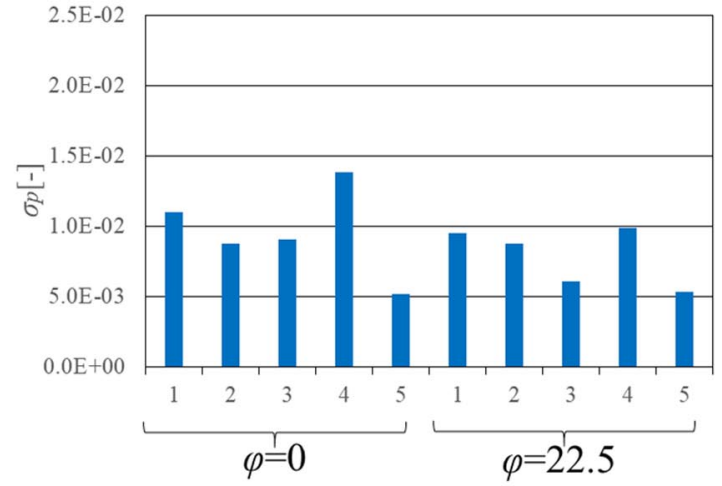

d) Case 2: interior

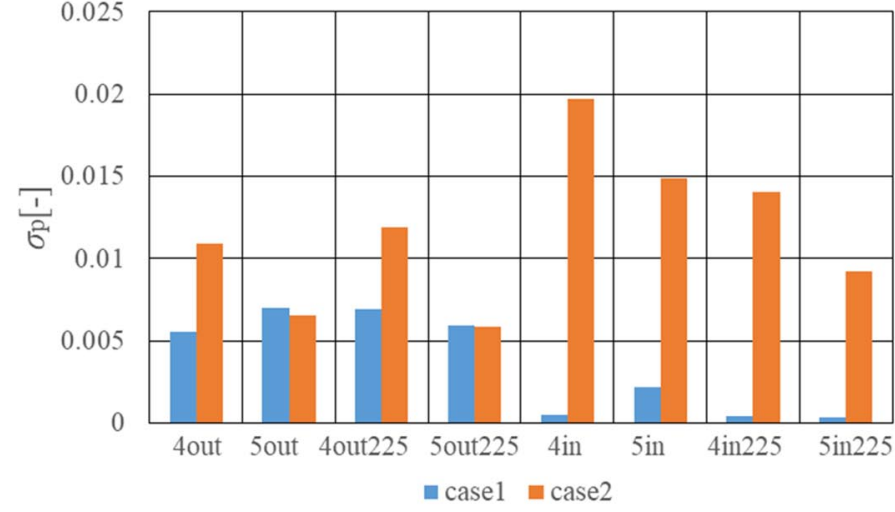

e) $\sigma_{p}$ of measurement points 4 and 5

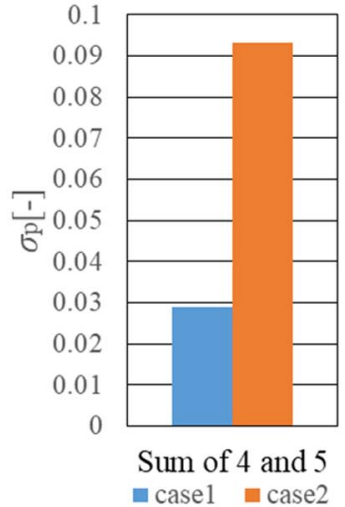

f) Sum of the $\sigma_{p}$ of measurement points 4 (interior and exterior) and 5 (interior and exterior)

Fig. $10 \sigma_{p}$ of each pressure measurement point.

(the schematic is shown in Fig. 13). The period of visualization $T$ in the figure is approximately $0.15 \mathrm{~s}(\cong 7 \mathrm{~Hz})$. We defined the time instant in Fig. $12 \mathrm{a}$ as $t / T=0.0$, and the results indicate that the pressure of 3 out 22.5 increases until $t / T=0.5$ because the reattachment region $R$ that indicates relatively high pressure gradually covers $3_{\text {out22.5 }}$. Subsequently, the pressure of $3_{\text {out22.5 }}$ decreases until $t / T=1.0$. As shown in case 1 's interior, the $\sigma_{p}$ of $1_{\text {out } 22.5}$ is the highest, although the details of the flowfield at this point are omitted because the fluctuation near the center of the parachute is not expected to play a role in the area oscillations.

\section{Case 2: Exterior}

Next, we focused on the exterior of case 2. Figure 14 shows a period of variation in the pressure distribution of $2_{\text {out } 0} \overline{\text { in }}$ case 2 . The period of visualization $\mathrm{T}$ in the figure is approximately $0.15 \mathrm{~s}$ $(\cong 7 \mathrm{~Hz}$ ). We defined the time in Fig. $14 \mathrm{a}$ as $t / T=0.0$ when the low-pressure region due to separation $S_{R}$ covers $2_{\text {out } 0}$. The results indicate that the pressure of $2_{\text {out } 0}$ increases until $t / T=0.5$ because relatively high-pressure region $R$ approaches to $2_{\text {out } 0}$. Subsequently, given the approach of $S_{R}$, the pressure of $2_{\text {out } 0}$ decreases again. In contrast to case 1, the reattachment region $\mathrm{R}$ and separated region $S_{R}$ move in the whole exterior region of case 2 (the schematic is shown in Fig. 15). This leads to the difference in the pressure and drag fluctuation amount between case 1 and case 2 . Additionally, Fig. 14 also shows the asymmetric pressure distribution of case 2's exterior. A band-support structure is absent in case 2, and thus $A_{V L}$ that restricts the movement of $\mathrm{R}$ in case 1 is not formed. Therefore, the results indicate that the reattachment region $\mathrm{R}$ can be gathered to the left side in this figure. In the case of a flexible parachute, this could potentially cause one-half of a canopy to deform if the pressure at both sides mutually interacts significantly. Figure 16a shows the $u$ velocity contours and velocity vectors of the flowfield near 


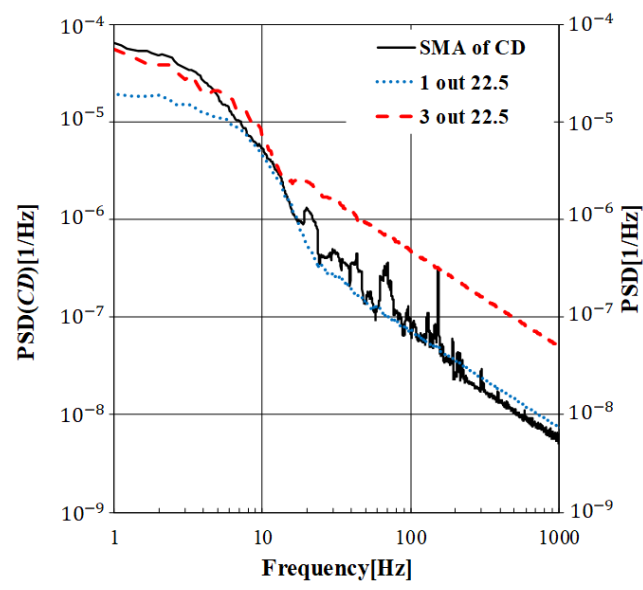

a) Case 1

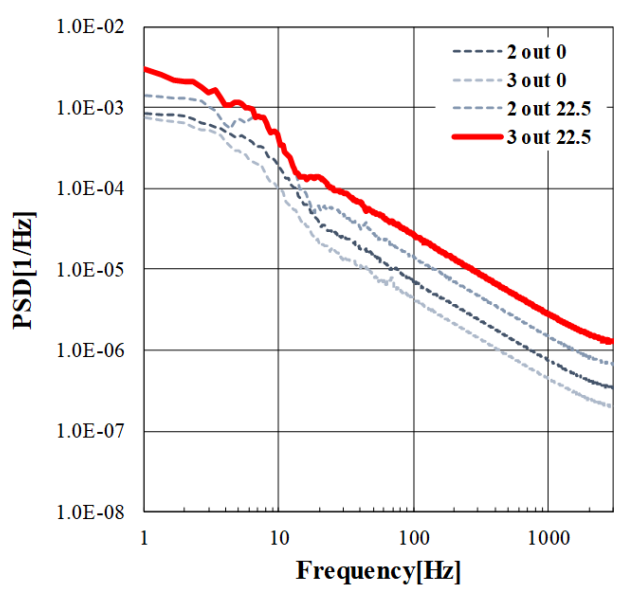

c) Case 1: PSD graph near $3_{\text {out } 22.5}$

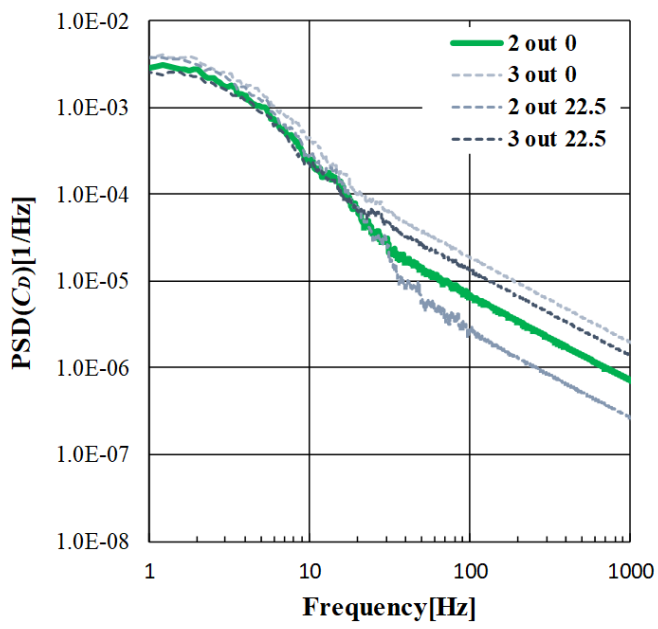

e) Case 2: PSD graph near $2_{\text {out }} 0$

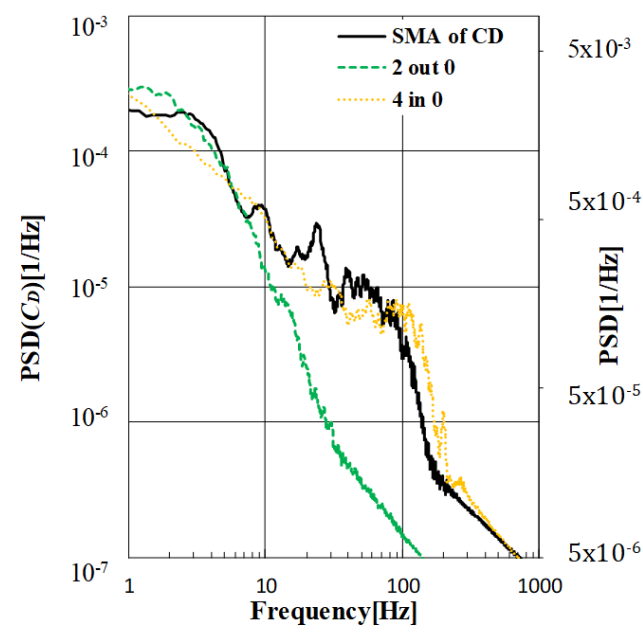

b) Case 2

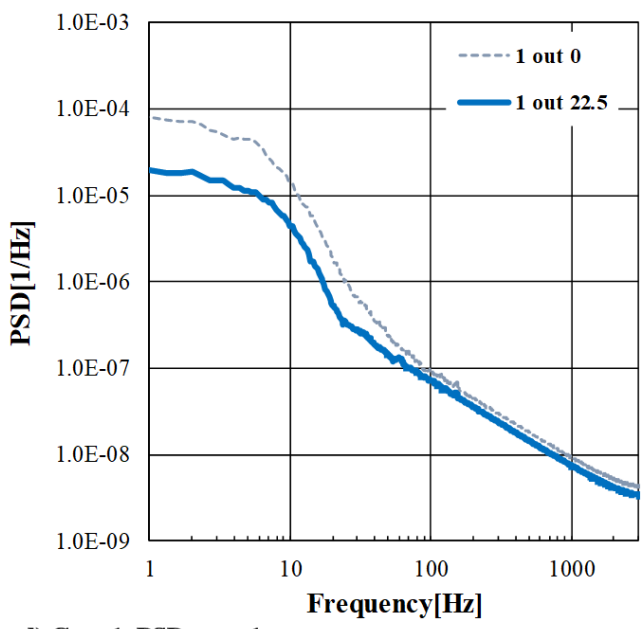

d) Case 1: PSD near $1_{\text {out } 22.5}$

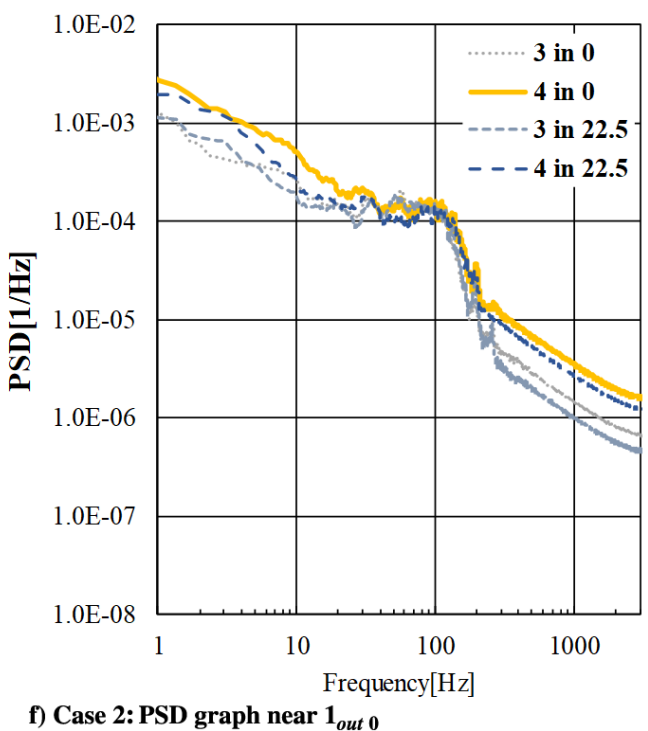

Fig. 11 Relation between surface pressure and drag coefficient $\left(C_{D}\right)$ fluctuation.

the reattachment region $\mathrm{R}$ at $t / T=0.0$ in case 1 [this plane is $\left.y /\left(D_{p} / 2\right)=0.55\right]$. The region colored in red represents the reverse flow region, and the reverse flow goes to reattachment region $\mathrm{R}$. Similarly, Fig. $16 \mathrm{~b}$ shows the $u$ velocity contours and velocity vectors of the flowfield around $R$ at $t / T=1.0$ in case 2 . As shown in the figure, it is observed that the reverse flow goes toward $R$. On the other side of R (right side in this figure), separation and vortex regions are observed.

\section{Case 2: Interior}

Finally, we focus on the interior of case 2. Figure 17 shows a single period of variation in the pressure distribution of $4_{\text {in } 0}$ in case 2 . 


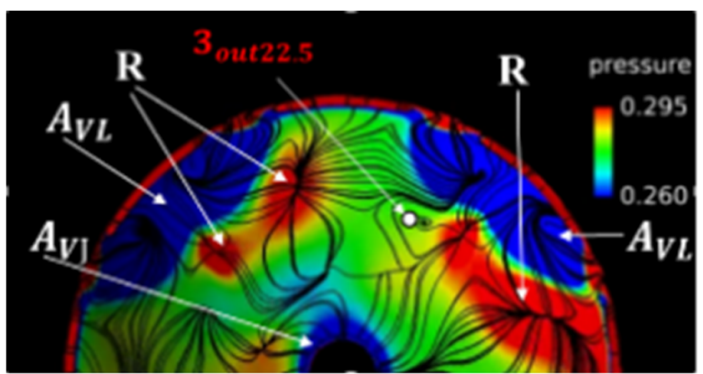

a) $\mathrm{t} / \mathrm{T}=\mathbf{0 . 0}$

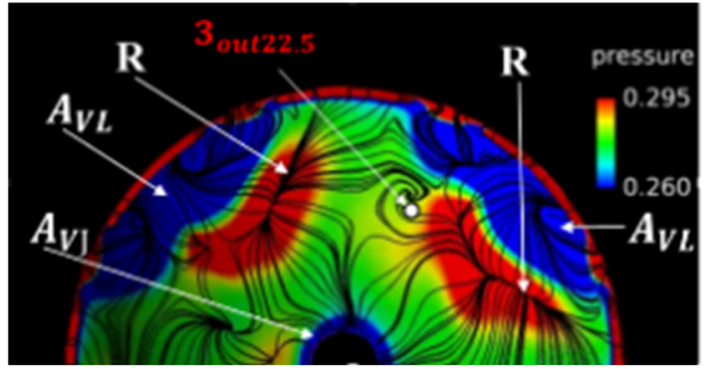

b) $\mathbf{t} / \mathbf{T}=\mathbf{0 . 2 5}$

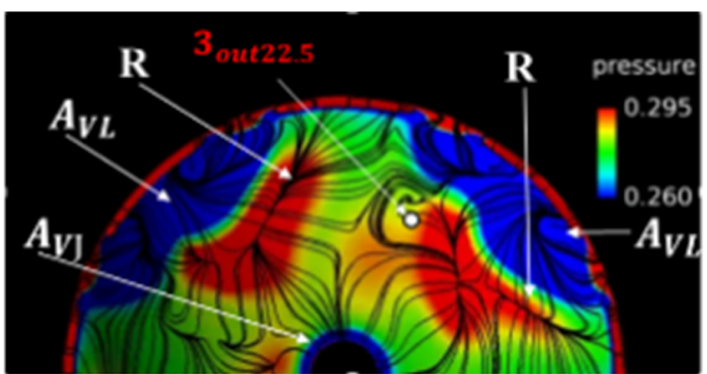

c) $\mathbf{t} / \mathbf{T}=0.5$

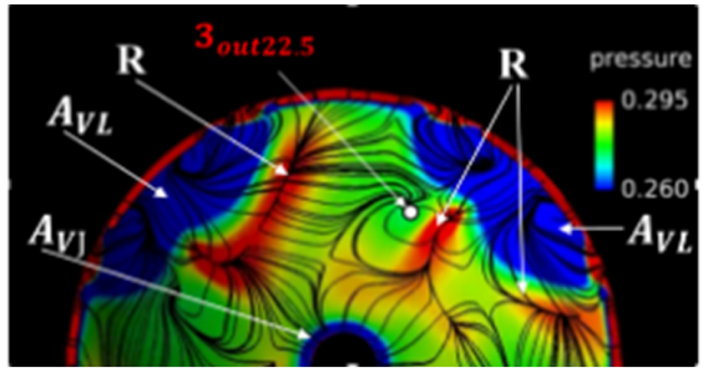

d) $\mathbf{t} / \mathbf{T}=0.75$

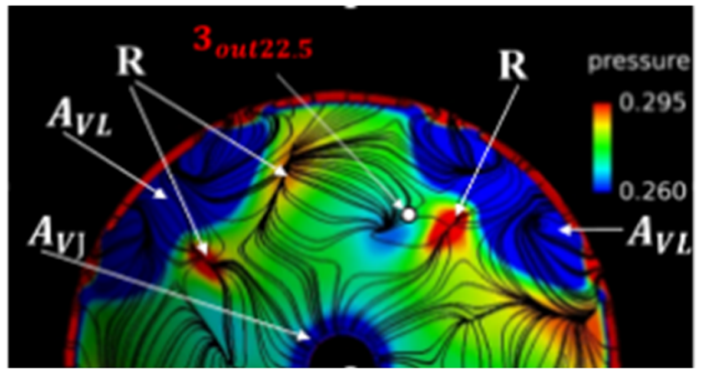

e) $\mathbf{t} / \mathbf{T}=\mathbf{1 . 0}$

Fig. 12 Pressure (normalized by the freestream value) distribution variation in case 1 .

The period of visualization $T$ in the figure is approximately $0.04 \mathrm{~s}$ $(25 \mathrm{~Hz})$, and we defined the time in Fig. $17 \mathrm{a}$ as $t / T=0.0 \mathrm{~s}$. Figure 18 shows the contours of the pressure and $y$ directional (see Fig. $\overline{3}$ ) velocity $v$ vectors at $t / T=0.0,0.5$, and 1.0 . When $t / T=0.0$, the

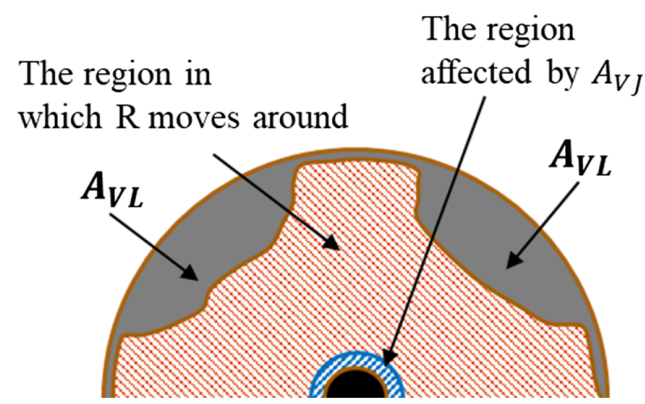

Fig. 13 Schematic of the area in which $R$ moves around (case 1).

stagnation regions $\left(S_{t}\right)$ gather to the lower-half in Fig. 17a, and the upper-half (left-half in Fig. 18a) corresponds to the vortex region. The pressure of $4_{\text {in } 0}$ decreases until $t / T=0.5$ when the expansion flow ("Exp.") becomes stronger (see Fig. 18b). Subsequently, the pressure of $4_{\text {in }}$ increases along with that of the complete region interior of the canopy. It was considered that the increase was caused by the departure of the vortex region from the interior wall of the canopy (see Fig. 18c). As shown in the result, in case 2, its pressure distribution exhibits large asymmetry even interior, and this causes large canopy deformation and collapse similar to that of the exterior. In contrast, when a band-support structure exists, this could act as a type of rectifying plate and can create a slightly symmetrical flow and pressure distribution interior of the canopy.

\section{Conclusions}

In the study, the CFD calculations were conducted to investigate the basic flowfield of the supersonic parachute (DGB type) and the two configurations were compared, wherein a configuration mimicked the wind-tunnel test model that exhibits a discrete gap opening area (case 1), and the other configuration mimicked a simplified actual configuration that exhibits a continuous gap opening area (case 2). Then, the following conclusions were obtained:

1) In terms of the amount of $C_{D}$ fluctuation, the results indicated that case 1 exhibited $60 \%$ less $C_{D}$ fluctuation than that in case 2 . The result suggested that the drag fluctuation can be underestimated by using a general wind-tunnel test model.

2) In terms of the pressure fluctuation amount, in case 1 , the fluctuation amount of the exterior was five times that of the interior. Thus, the fluctuation in the exterior was dominant because all of the fluctuation was generated by the interaction between jets from the gap or the vent and wake of the canopy. Additionally, the results indicated that the interaction generated the reattachment region on the exterior surface and the moving of the region caused the main drag fluctuation.

3) Conversely, in case 2, the fluctuation amount of the exterior was only 1.2 times that of the interior. This was because the interior also exhibits large asymmetry pressure distribution due to the lack of band-support structure. A comparison with the pressure distribution in case 1 indicated that the structure suppressed the exterior pressure fluctuation. The structure generated a vortex region that caused a relatively small pressure fluctuation backward and restricted the movement of the reattachment region, causing a high-pressure fluctuation. Without the structure, the amount of outflow from gap in each direction was gathered to a side, and its asymmetry produced large fluctuations. As described earlier in this paper, in case 2, large fluctuations occurred both interior and exterior, given that the benefit of the structure was absent.

4) From the area oscillation suppression viewpoint, case 1 was considered as a better configuration. This was because the area oscillation occurred when the position far from the parachute center was deformed; and the effect of the band-support structure, as previously described, reduced $70 \%$ of the pressure fluctuation.

5) In case 1, as shown in the PSD graph and visualization of the pressure distribution, the fluctuation of less than $15 \mathrm{~Hz}$ was considered to mainly occur at the position corresponding to approximately $60 \%$ of the radius from the center of the parachute. Additionally, the 


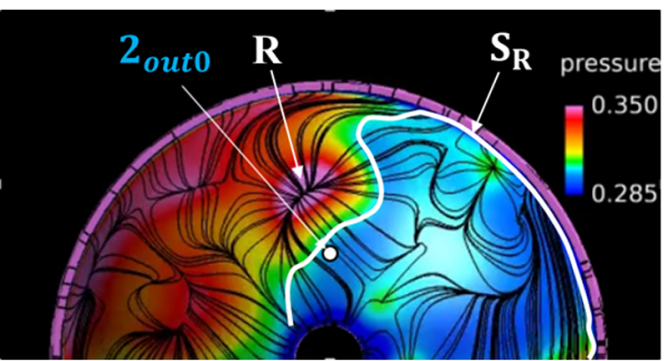

a) $t / T=0.0$

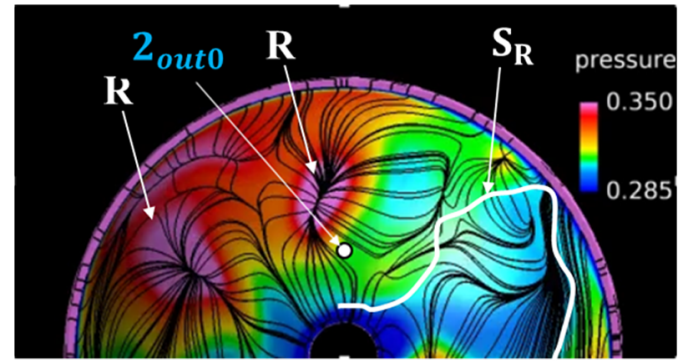

b) $\mathbf{t} / \mathbf{T}=\mathbf{0 . 2 5}$

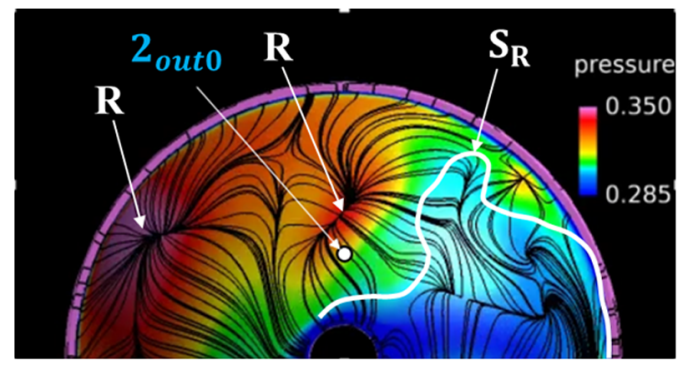

c) $\mathrm{t} / \mathrm{T}=0.5$

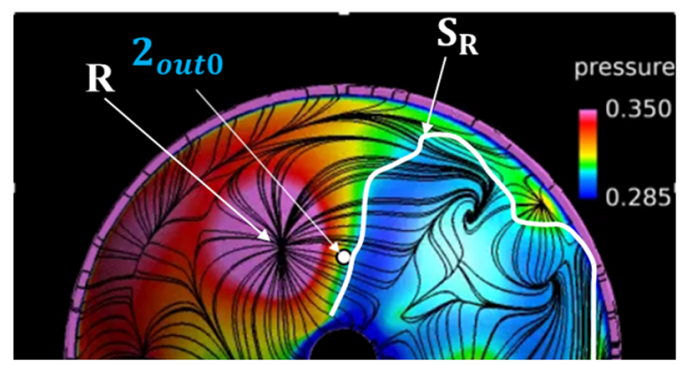

d) $\mathbf{t} / \mathbf{T}=\mathbf{0 . 7 5}$

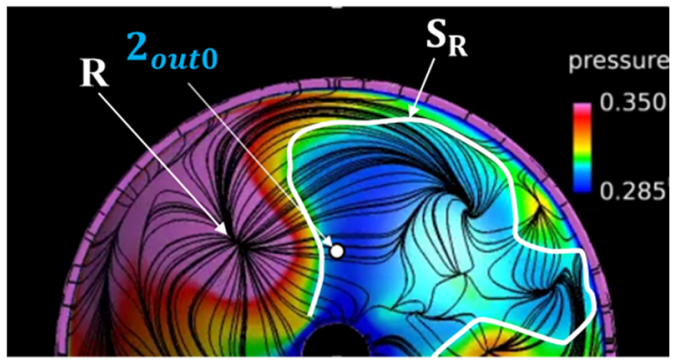

e) $\mathbf{t} / \mathbf{T}=\mathbf{1 . 0}$

Fig. 14 Pressure (normalized by the freestream value) distribution variation in case 2 .

fluctuation exceeding $15 \mathrm{~Hz}$ was considered to mainly occur at the exterior near the vent.

6) Similarly, in case 2, the fluctuation of less than $9 \mathrm{~Hz}$ was considered to mainly occur at the position corresponding to approximately $40 \%$ of the radius from the center of the parachute. In

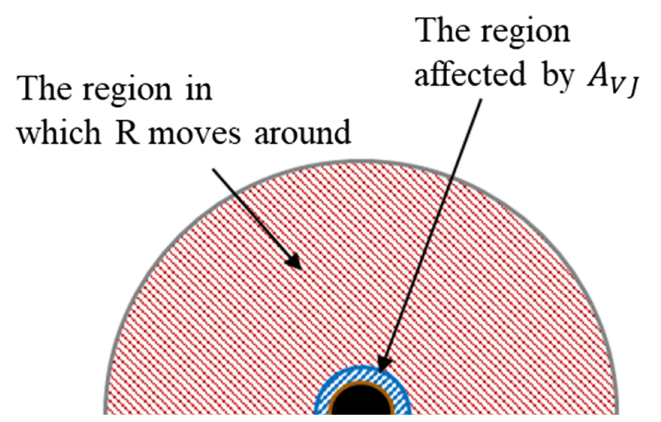

Fig. 15 Schematic of the area in which $R$ moves around (case 2).

contrast, the fluctuation in the range of $100-200 \mathrm{~Hz}$ was considered to mainly occur at the position corresponding to approximately $80 \%$ of the radius from the center of the parachute.

The present, fundamental work has been limited to the rigid, impermeable assumption on the canopy with a relatively large thickness, as well as the absence of the forebody or suspension lines, while geometrical porosities of the gap and vent have been considered. These will be taken into account in future studies for better explanations on the wake flows, their interaction with the canopy deformation, and the other detailed parts at higher frequencies. A higher Mach number or Reynolds number (i.e., larger geometries) will also provoke higherfrequency modes, calling for extra computational burdens; such a survey is also currently planned as a separate work, as well as a survey on the support effect in the wind-tunnel experiment. Taking all these into account will eventually lead to a good guideline for designing

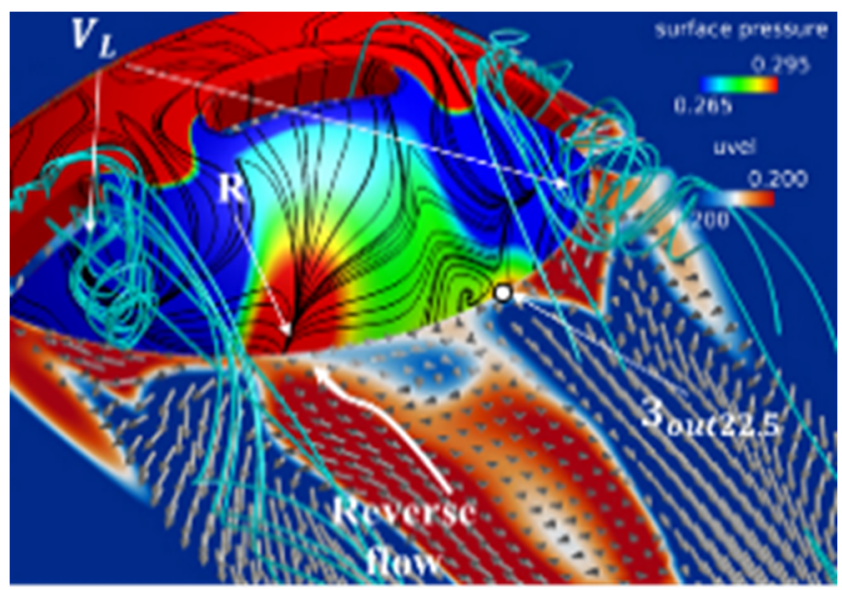

a) Case 1

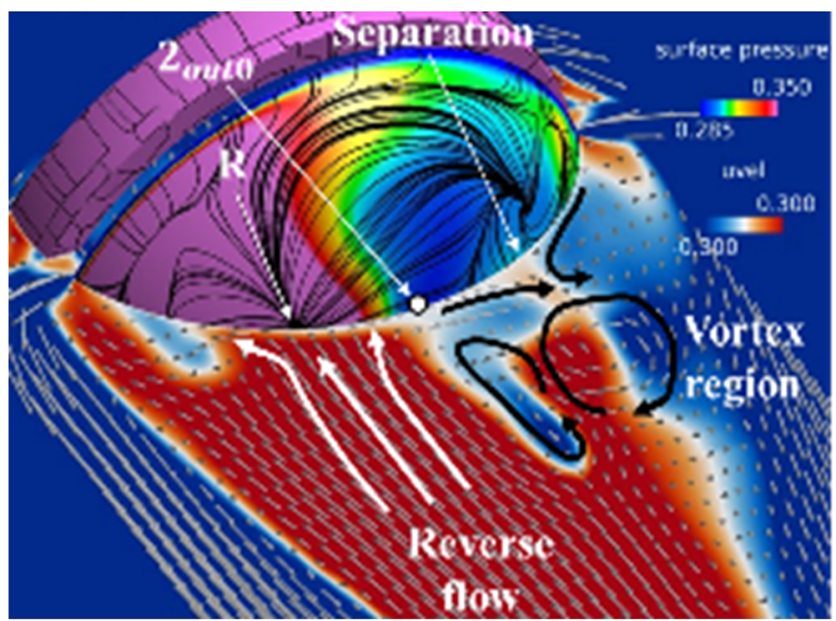

b) Case 2

Fig. 16 Reattachment region and surrounding flow. 


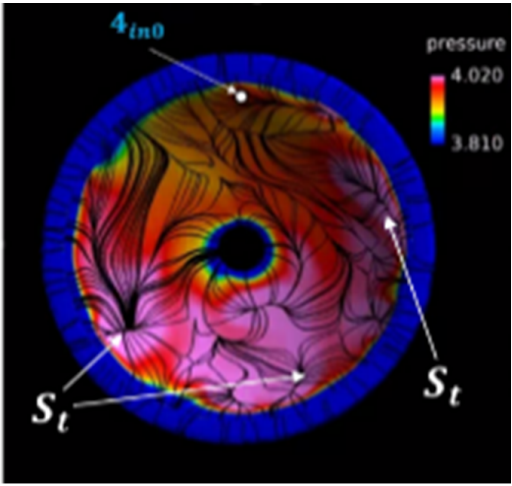

a) $\mathrm{t} / \mathrm{T}=\mathbf{0 . 0}$

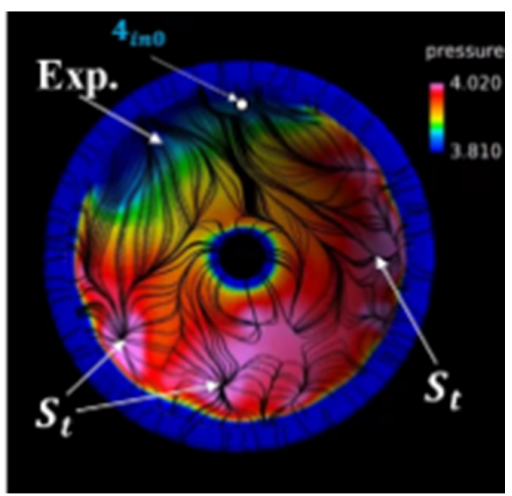

c) $\mathrm{t} / \mathrm{T}=0.5$

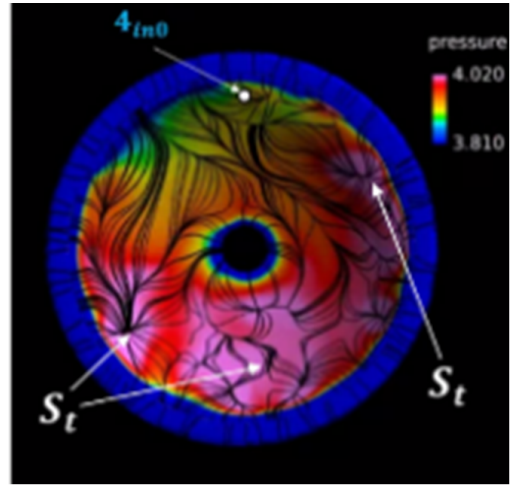

b) $t / T=0.25$

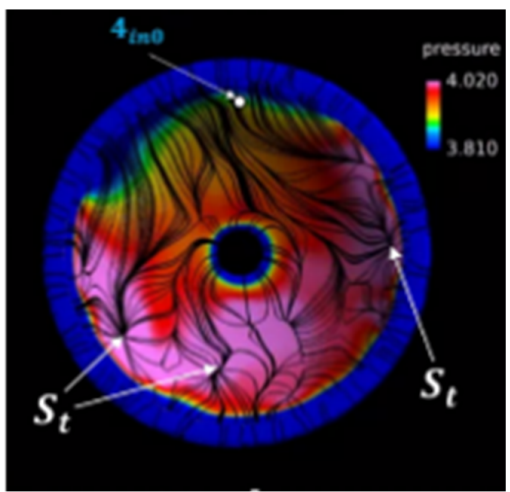

d) $t / T=0.75$

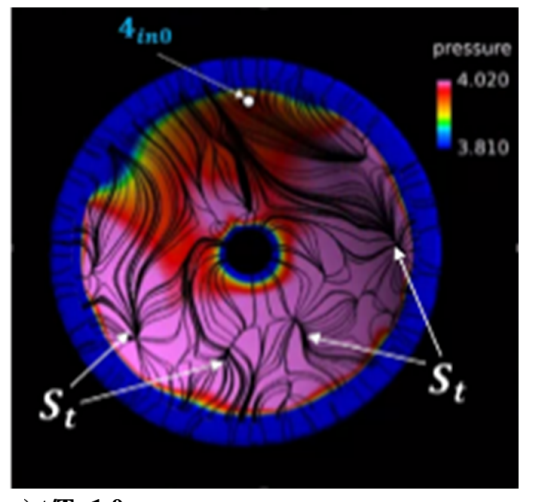

e) $t / T=1.0$

Fig. 17 Pressure (normalized by the freestream value) distribution variation for case 2 (interior).

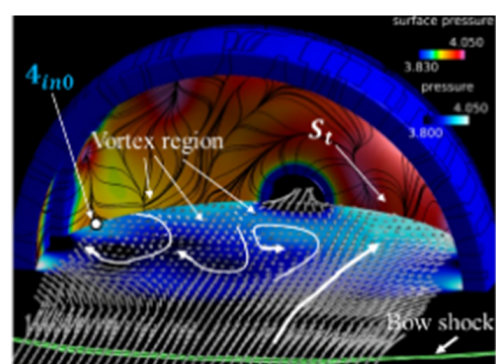

a) $t / T=0.0 \mathrm{~s}$

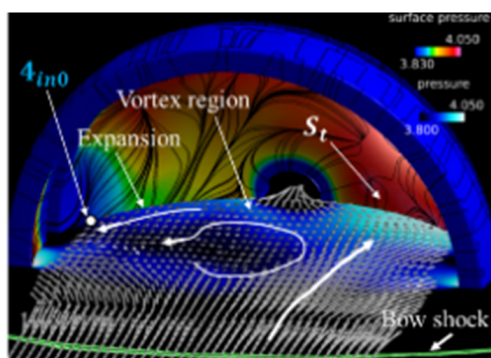

b) $t / T=0.5$

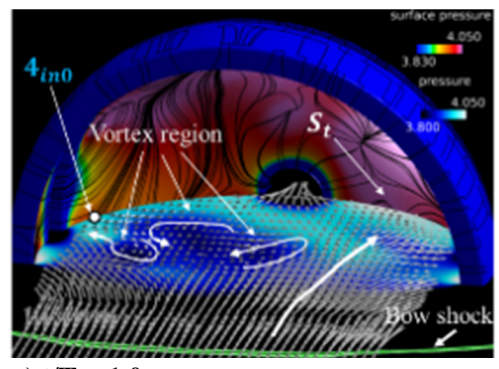

c) $t / T=1.0$

Fig. 18 Pressure (normalized by the freestream value) fluctuation and flowfield interior the canopy in case 2. 
novel parachute configurations and further developments of the related studies.

\section{Acknowledgments}

The authors used HexaGrid (a grid generation tool) and FaSTAR (numerical fluid solver), and they performed the calculations on the Japan Aerospace Exploration Agency (JAXA) generation 2 supercomputer system. The authors sincerely thank JAXA, Masato Taguchi at the National Defense Academy in Japan, and our laboratory members (specifically, Yuya Yasumura) for their cooperation.

\section{References}

[1] Cruz, J. R., and Lingard, J. S., "Aerodynamic Decelerators for Planetary Exploration: Past, Present, and Future," AIAA Guidance, Navigation, and Control Conference and Exhibit, AIAA Paper 2006-6792, 2006. https://doi.org/10.2514/6.2006-6792

[2] Maydew, R. C., Peterson, C. W., and Orlik-Ruckemann, K. J., "Design and Testing of High-Performance Parachutes," AGARDograph 319, NeuillySur-Seine, France, 1991, p. 12, https://www.sto.nato.int/publications/ AGARD/AGARD-AG-319/AGARD-AG-319.pdf [retrieved 29 July 2020].

[3] Reichenau, D. E., "Aerodynamic Characteristics of Disk-Gap-Band Parachutes in the Wake of Viking Entry Forebodies at Mach Numbers from 0.2 to 2.6," Arnold Engineering Development Center AEDC-TR72-78, Arnold AFB, TN, 1972, https://apps.dtic.mil/dtic/tr/fulltext/u2/ 746291.pdf [retrieved 29 July 2020].

[4] Sengupta, A., Steltzner, A., Witkowski, A., Candler, G., and Pantano, C., "Findings from the Supersonic Qualification Program of the Mars Science Laboratory Parachute System," AIAA Aerodynamic Decelerator Systems Conference, AIAA Paper 2009-2900, 2009. https://doi.org/10.2514/6.2009-2900

[5] Semba, N., Kuzuo, K., Taguchi, M., and Mori, K., "Statistical Characteristics of the Pressure Oscillation in the Canopy of Supersonic Parachute," Journal of the Society for Aeronautical and Space Sciences, Vol. 65, No. 2, 2017, pp. 64-72 (in Japanese).

[6] Karagiozis, K., Kamakoti, R., Cirak, F., and Pantano, C., "A Computational Study of Supersonic Disk-Gap-Band Parachutes Using LargeEddy Simulation Coupled to a Structural Membrane," Journal of Fluids and Structures, Vol. 27, No. 2, 2011, pp. 175-192. https://doi.org/10.1016/j.jfluidstructs.2010.11.007

[7] Sengupta, A., Roeder, J., Kelsch, R., Wernet, M., Kandis, M., and Witkowski, A., "Supersonic Disk Gap Band Parachute Performance in the Wake of a Viking-Type Aeroshell from Mach 2 to 2.5," AIAA Paper 2008-6217, 2008. https://doi.org/10.2514/6.2008-6217

[8] Kim, Y., and Peskin, C. S., "3-D Parachute Simulation by the Immersed Boundary Method," Computers and Fluids, Vol. 38, No. 6, 2009, pp. 1080-1090. https://doi.org/10.1016/j.compfluid.2008.11.002

[9] Xue, X., and Nakamura, Y., "Numerical Simulation of a ThreeDimensional Flexible Parachute System Under Supersonic Conditions," Transactions of the Japan Society for Aeronautical and Space Sciences, Aerospace Technology Japan, Vol. 11, Nov. 2013, pp. 99-108. https://doi.org/10.2322/tastj.11.99

[10] Xue, X., Koyama, H., Nakamura, Y., and Wen, C.-Y., "Effects of Suspension Line on Flow Field Around a Supersonic Parachute," Aerospace Science and Technology, Vol. 43, June 2015, pp. 63-70. https://doi.org/10.1016/j.ast.2015.02.014

[11] Xue, X.-P., Nishiyama, Y., Nakamura, Y., Mori, K., and Wen, C.-Y., "Parametric Study on Aerodynamic Interaction of Supersonic Parachute System," AIAA Journal, Vol. 53, No. 9, 2015, pp. 2796-2801. https://doi.org/10.2514/1.J053824

[12] Guruswamy, G. P., "Time-Accurate Coupling of Three-Degree-ofFreedom Parachute System with Navier-Stokes Equations," Journal of Spacecraft and Rockets, Vol. 54, No. 6, 2017, pp. 1278-1283. https://doi.org/10.2514/1.A33835

[13] Tezduyar, T. E., Takizawa, K., Moorman, C., Wright, S., and Christopher, J., "Space-Time Finite Element Computation of Complex FluidStructure Interactions," International Journal for Numerical Methods in Fluids, Vol. 64, Nos. 10-12, 2010, pp. 1201-1218. https://doi.org/10.1002/fld.2221

[14] Stein, K., Benney, R., Kalro, V., Tezduyar, T. E., Leonard, J., and Accorsi, M., "Parachute Fluid-Structure Interactions: 3-D Computation," Computer Methods in Applied Mechanics and Engineering, Vol. 190, Nos. 3-4,
2000, pp. 383-386.

https://doi.org/10.1016/S0045-7825(00)00208-5

[15] "Performance of and Design Criteria for Deployable Aerodynamic Decelerators," U.S. Air Force ASD-TR-61-579, Dec. 1963, https:// apps.dtic.mil/dtic/tr/fulltext/u2/429971.pdf [retrieved 29 July 2020].

[16] Heinrich, H. G., Ballinger, J. G., and Ryan, P. E., "Pressure Distribution in Transonic Flow of Ribbon and Guide Surface Parachute Models," U.S. Air Force WADC 59-32, Feb. 1959. https://apps.dtic.mil/dtic/tr/ fulltext/u2/210257.pdf [retrieved 29 July 2020].

[17] Fric, T. F., and Roshko, A., "Vortical Structure in the Wake of a Transverse Jet," Journal of Fluid Mechanics, Vol. 279, Nov. 1994, pp. $1-47$. https://doi.org/10.1017/S0022112094003800

[18] Aswin, G., and Chakraborty, D., "Numerical Simulation of Transverse Side Jet Interaction with Supersonic Free Stream," Aerospace Science and Technology, Vol. 14, No. 5, 2010, pp. 295-301. https://doi.org/10.1016/j.ast.2010.02.001

[19] Takayanagi, H., Suzuki, T., Yamada, K., Maru, Y., Matsuyama, S., and Fujita, K., "Development of Supersonic Parachute for Japanese Mars Rover Mission," Transactions of the Japan Society for Aeronautical and Space Sciences, Aerospace Technology Japan, Vol. 14, No. ists30, 2016, pp. Pe_87-Pe_94.

https://doi.org/10.2322/tastj.14.Pe_87

[20] Spalart, P. R., Deck, S., Shuur, M. I., Squires, K. D., Strelets, M. K., and Travin, A., "A New Version of Detached-Eddy Simulation, Resistant to Ambiguous Grid Densities," Theoretical and Computational Fluid Dynamics, Vol. 20, No. 3, 2006, pp. 181-195. https://doi.org/10.1007/s00162-006-0015-0

[21] Pain, R., Weiss, P.-E., and Deck, S., "Zonal Detached Eddy Simulation of the Flow Around a Simplified Launcher Afterbody," AIAA Journal, Vol. 52, No. 9, 2014, pp. 1967-1979. https://doi.org/10.2514/1.J052743

[22] Kuzuo, K., Taguchi, M., Kurata, R., Arihama, K., and Mori, K., "Shape Effect on the Aerodynamics of Supersonic Parachute," Transactions of the Japan Society for Aeronautical and Space Sciences (in preparation).

[23] Cruz, J. R., Way, D., Shidner, J., Davis, J. L., Powell, R. W., Kipp, D., Adams, D. S., Sengupta, A., Witowski, A., and Kandis, M., "Parachute Models Used in the Mars Science Laboratory Entry, Descent, and Landing Simulation," AIAA Aerodynamic Decelerator Systems Technology Conference, AIAA Paper 2013-1276, 2013. https://doi.org/10.2514/6.2013-1276

[24] Hashimoto, A., Murakami, K., Aoyama, T., Hishida, M., Paulus, R. L., Sakashita, M., and Sato, Y., "Development of Fast Flow Solver FaSTAR," Journal of the Society for Aeronautical and Space Sciences, Vol. 63, No. 3, 2015, pp. 96-105 (in Japanese). https://doi.org/10.2322/jisass.63.96

[25] Shima, E., and Kitamura, K., "Parameter-Free Simple Low-Dissipation AUSM-Family Scheme for All Speeds," AIAA Journal, Vol. 49, No. 8, 2011, pp. 1693-1709. https://doi.org/10.2514/1.J050905

[26] Shima, E., Kitamura, K., and Haga, T., "Green-Gauss/Weighted-LeastSquares Hybrid Gradient Reconstruction for Arbitrary Polyhedra Unstructured Grids," AIAA Journal, Vol. 51, No. 11, 2013, pp. 2740 2747.

https://doi.org/10.2514/1.J052095

[27] Venkatakrishnan, V., "Convergence to Steady State Solutions of the Euler Equations on Unstructured Grids with Limiters," Journal of Computational Physics, Vol. 118, No. 1, 1995, pp. 120-130. https://doi.org/10.1006/jcph.1995.1084

[28] Jameson, A., and Turkel, E., "Implicit Schemes and LU Decompositions," Mathematics of Computation, Vol. 37, No. 156, 1981, pp. 385397. https://doi.org/10.1090/S0025-5718-1981-0628702-9

[29] Hashimoto, A., Murakami, K., Aoyama, T., Yamamoto, K., Murayama, M., and Lahur, P. R., "Drag Prediction on NASA CRM Using Automatic Hexahedra Grid Generation Method," Journal of Aircraft, Vol. 51, No. 4, 2014, pp. 1172-1182. https://doi.org/10.2514/1.C032641

[30] Takayanagi, H., Yamada, K., Maru, Y., and Fujita, K., "Development of Supersonic Parachute for Mars EDL Demonstrator," Proceedings of the 58th Space Sciences and Technology Conference, Japan Soc. for Aeronautical and Space Sciences (JSASS) Paper JSASS-2014-4168, 2014 (in Japanese). 